\title{
52. SILICIFIED SEDIMENTS AND SILICA DIAGENESIS IN THE GOBAN SPUR AREA OF THE NORTHEAST ATLANTIC, LEG 80 ${ }^{1}$
}

\author{
Kenichi Otsuka, Institute of Geosciences, Faculty of Science, Shizuoka University ${ }^{2}$
}

\begin{abstract}
X-ray powder diffraction and optical and scanning-electron microscope analyses of sediment samples taken from four sites drilled in the Goban Spur area of the northeast Atlantic show variable diagenetic silicification of sediments at several stratigraphic horizons. The results are as follows:

1. The silicified sediments are middle Eocene at Site 548, Paleocene to lower Albian at Site 549, upper to lower Paleocene at Site 550, and lower Turonian at Site 551.

2. There are three types of these silicified sediments: nodular type in carbonate-rich host sediments, bedded type in clayey host sediments, and a type transitional between the other two.

3. Silica diagenesis is considered to progress as follows: dissolution of siliceous fossils; precipitation of opal CT in pore spaces and transformation of biogenic silica (opal A) to opal CT; development of opal CT cement; chalcedonic quartz precipitation in pore spaces and replacement of foraminiferal tests by chalcedonic quartz; and finally, transformation of opal CT to quartz, and cementation. But the strong influence of host-sediment types on diagenetic silica facies is recognized. Bedded-type silicified sediments in a clayey environment indicate a lower grade of silica diagenesis. Only very weak chalcedonic quartz formation is recognized, and there is no opal CT cementation, even in Lower Cretaceous bedded-type clayey silicified sediments.

4. The $d(101)$ spacing of opal CT shows two distinct trends of ordering or decrease with burial depth; one is a rapid change, in the case of nodular silicified sediments, and the other is a more gentle shift, found in bedded silicified sediments.

5. Diagenetic silica facies of the nodular type develop as irregular concentric zones around some nodule nuclei. Also, quartz-chert nodule formation occurs at rather shallower horizons, and is discordant with the trend of decreasing $d(101)$ spacing in opal CT.

6. Silicified sediments at Site 551 are shallower than at the other sites. The diagenetic silica facies suggest the probable erosion of $300 \mathrm{~m}$ or more of sediment at this site.

7. The zeolites clinoptilolite and phillipsite were found in the sediment samples recovered on Leg 80 . Clinoptilolite occurs from the shallower levels to the deepest horizons of diagenetically silicified zones, suggesting that clinoptilolite formation is related to diagenesis of biogenic silica. Phillipsite at Site 551 (Section 551-5-2) may originate from volcanogenic material.
\end{abstract}

\section{INTRODUCTION}

Detailed studies of silicified sediments from DSDP samples have clarified the worldwide distribution of cherts and other silicified sediments in ocean basins. These studies have yielded many new insights regarding the occurrence and the nature of various silicified sediments. They have also revealed very diverse features of silica diagenesis and the formation of cherts and other siliceous sediments, such as porcellanites and diatomites (Heath, 1973; Garrison et al., 1975; Keene, 1975; Kelts, 1976; Kagami, 1979; Pisciotto, 1980; Riech, 1980; Grehin et al., 1981; Hein et al., 1981; Riech, 1981).

Previous works have identified three main diagenetic silica mineral facies in these silicified sediments: (1) $\mathrm{X}$-ray-amorphous silica (opal A), (2) disordered $\alpha$-cristobalite (opal CT), and (3) stable quartz (Jones and Segnit, 1971). Also, silicified sediments can be classified into two major groups: a bedded type occurring in clayey or siliceous host sediments, and a nodular type found in predominantly calcareous host sediments (Heath and Moberly, 1971; Lancelot, 1973; von Rad et al., 1978;

\footnotetext{
${ }^{1}$ Graciansky, P. C. de, Poag, C. W., et al., Init. Repts. DSDP, 80: Washington (U.S Govt, Printing Office)

2 Address: Inst. of Geosciences, Faculty of Science, Shizuoka Univ., Japan.
}

Riech and von Rad, 1979). The bedded type in clayey or siliceous sediments has a tendency to change into socalled opal-CT porcellanites, whereas the nodular type in carbonate-rich sediments tends to be composed of quartz-cherts. Thus, the sources, processes, and other factors influencing the silica diagenesis of these two types in different host sediment facies may be different. Lancelot (1973) presented a quartz-precipitation theory based on observation of these siliceous minerals. He postulated that the different micro-environments of the host sediments control the diagenetic processes and the resultant products, and that even quartz is able to precipitate directly in a carbonate-predominant porous matrix. The diagenetic-transformation theory of silica minerals is another theory on the formation of silicified sediments, and seems to be generally accepted. Riech and von Rad (1979a) described silica diagenesis as the maturation of silica mineral from biogenic amorphous silica (opal A) to opal CT, further to quartz by dissolution-precipitation processes and/or quasi-solid-solid microstructural conversion.

These transformations of silica minerals do not always show straightforward progress, however, and the field evidence indicates very irregular occurrences in many cases. Silicic zeolites and other silicates such as clay minerals are sometimes closely related to the silica-mineral 
diagenesis and modes of occurrence (Riech, 1979; Iijima et al., 1980). Volcanogenic silica sources may be important in some cases.

During Leg 80 of the Deep Sea Drilling Project, four sites were drilled (Sites 548, 549, 550, and 551). Each site contains zones of silicified sediments in rather limited stratigraphic intervals. A total of 414 samples (45 from Site 548, 156 from Site 549, 179 from Site 550, and 34 from Site 551), ranging from Pleistocene to Barremian, were studied by X-ray diffraction analysis. Fortyfour selected samples were further investigated by thinsection examination under the optical microscope. Some samples were also investigated by scanning-electron microscope.

This chapter summarizes the results of the mineralogical and petrological analyses of the silicified sediments sampled from the four sites of Leg 80 .

\section{METHODS AND TERMS}

Qualitative XRD analyses were performed on all samples, both onboard Glomar Challenger and in the laboratory, as already mentioned. Semiquantitative X-ray diffraction analyses were also carried out according to the method of Mitsui (1975). Values of the $d(101)$ spacing of opal CT in silicified sediments were calculated by precise XRD, using silicon as the standard material and under the condition of slow $2 \theta$-speed $\left(1 / 4^{\circ}\right.$ per min.). Obstacle feldspars are very scarce in Leg 80 samples in general, so the XRD peak at $22^{\circ}(\mathrm{CuK} \alpha)$ is considered to be attributable almost solely to opal CT.

Diagenetic quartz was distinguished from clastic quartz grains by its characteristics in the matrix, such as its grain form, its texture (as in chalcedony aggregate), and its relation to fossil tests under the optical microscope; then its abundance was estimated mainly by visual means under the microscope, referring to XRD estimates and the background quartz contents of host sediments.

The classification and terminology of silicified sediments, designating sediments containing diagenetic silica constituents, is here based on the method proposed by von Rad et al. (1978). Amounts of three silica-mineral polymorphs-opal A, opal CT, and diagenetic quartzwere estimated by thin-section and XRD analyses, and the sediments were classified as follows (Fig. 1 legend):

1. Immature, weakly silicified sediments, containing less than $50 \%$ diagenetic silica minerals, in which opal CT predominates over quartz.

2. Porcellanite, containing more than $50 \%$ diagenetic silica, with opal CT predominant over diagenetic quartz.

3. Mature quartz-chert, containing more than $50 \%$ diagenetic quartz with the quartz heavily predominant over opal CT.

\section{DISTRIBUTION AND OCCURRENCES OF SILICIFIED SEDIMENTS}

Silicified sediments were recovered from all Leg 80 sites, but their lithofacies and occurrences vary, as do their ages, which range from 21 m.y. (lower Miocene) to $106 \mathrm{~m} . y$. (lower Albian). Three types of silicified sediments are recognized in Leg 80 samples. They are a bedded type in clayey host sediments, a nodular type in calcareous host sediments, and a transitional type in both sediments.

Sediments encountered at all Leg 80 sites are more or less calcareous, composed of foraminiferal and nannofossil oozes or chalks. Nodular silicified sediments therefore occur at all sites. Excepting Site 551, their sub-bottom depths of occurrence coincide fairly well $(350-440 \mathrm{~m})$, despite differences in ages of host sediments (Fig. 1). The bedded silicified sediments have a relatively limited occurrence. Calcareous siltstones from Cores 549-32 to 549-48 (sub-bottom depth 483.5-626.0 m, Unit 6) con- tain a considerable amount of siliceous organic remains which have been transformed to opal CT. These lower to middle Albian occurrences are the oldest and deepest bedded-type silicified sediments encountered on Leg 80 . Clinoptilolite at Leg 80 sites has a more extensive stratigraphic range than the silicified sediments.

\section{Site 548}

Silicified sediments recovered from Site 548 are all of the hard nodular type; the nodules are pebble size and grayish green $(10 \mathrm{GY} 5 / 2)$ to dusky yellow green (5GT $5 / 2$ ) or gray (N3). The host sediments are middle Eocene pale grayish green (5G 8/1-5GY 7/1), firm bioturbated nannofossil chalk. They occur in lithologic Subunit $4 \mathrm{~b}$, from Section 548A-19-4 to Section 548A-21,CC (sub-bottom depth 382.2-401.5 m). Most siliceous remains found in this interval are radiolarians, although a few sponge spicules are present. Clinoptilolite occurrences span a wider range than the diagenetic silica minerals, from Section 548A-14-2 to Section 548A-22-7 (middle Miocene to middle Eocene).

\section{Site 549}

Various types of silicified sediments were recovered at Site 549. They show a very wide range of ages, sub-bottom depths, and types of occurrence (from Section 549-18-1, upper Paleocene, sub-bottom depth $350.7 \mathrm{~m}$, to Section 549-48-1, lower Albian, sub-bottom depth $626.3 \mathrm{~m}$ ). These sediments correspond to sediment Subunit $3 \mathrm{~d}$ and Units 4,5 , and 6 . Subunit $3 \mathrm{~d}$ and Units 4 and 5 are predominantly nannofossil chalks. Silicified sediments sparsely present in these chalk environments are generally of the nodular type, although some may be transitional to the bedded type.

Section 549-18-1 contains silicified sediments in the intervals $10-35 \mathrm{~cm}$ and $70-90 \mathrm{~cm}$ as layers of light brownish gray (10YR 6/2) fragmented hard breccias. These layers may be classified as the transitional type between bedded and nodular porcellanite. The host sediments are gray to light brownish gray siliceous nannofossil chalk of sediment Subunit 3d, bearing considerable amounts of sponge spicules, radiolarians, and diatoms. Sediment Unit 4 (light-colored nannofossil chalks) contains reddish brown (5YR 4/4) to olive-yellow (5Y 6/6) silicified nodules at 549-24-2, 55-65 cm; 549-24-3, 0-5 cm; $549-25-2,50-55 \mathrm{~cm}$; 549-25-3, 0-10 cm; and 549-26-1, $0-10 \mathrm{~cm}$. These nodules indicate a higher maturity of silica-mineral composition. For example, a nodule from 549-24-3, 0-10 cm is composed of quartz as the only silica mineral, and shows a mature quartz-chert texture. Silicified nodular sediments were also recovered from the carbonaceous black shale of Unit 5 in 549-27-1, 24-30 cm, and from the top of Core 549-32.

The calcareous siltstone layers of sediment Unit 6 (from Cores 549-32 to 549-48) are almost uniform in lithofacies, and form bedded-type silicified sediment strata through the unit, in which the maturity of silica diagenesis is fairly low. In spite of its considerable thickness (sub-bottom depth $483.5-626.3 \mathrm{~m}$ ), the sediment is restricted to middle to lower Albian. These weakly silicified beds are gray (N4-N8) to greenish gray (5GY 4/1) 

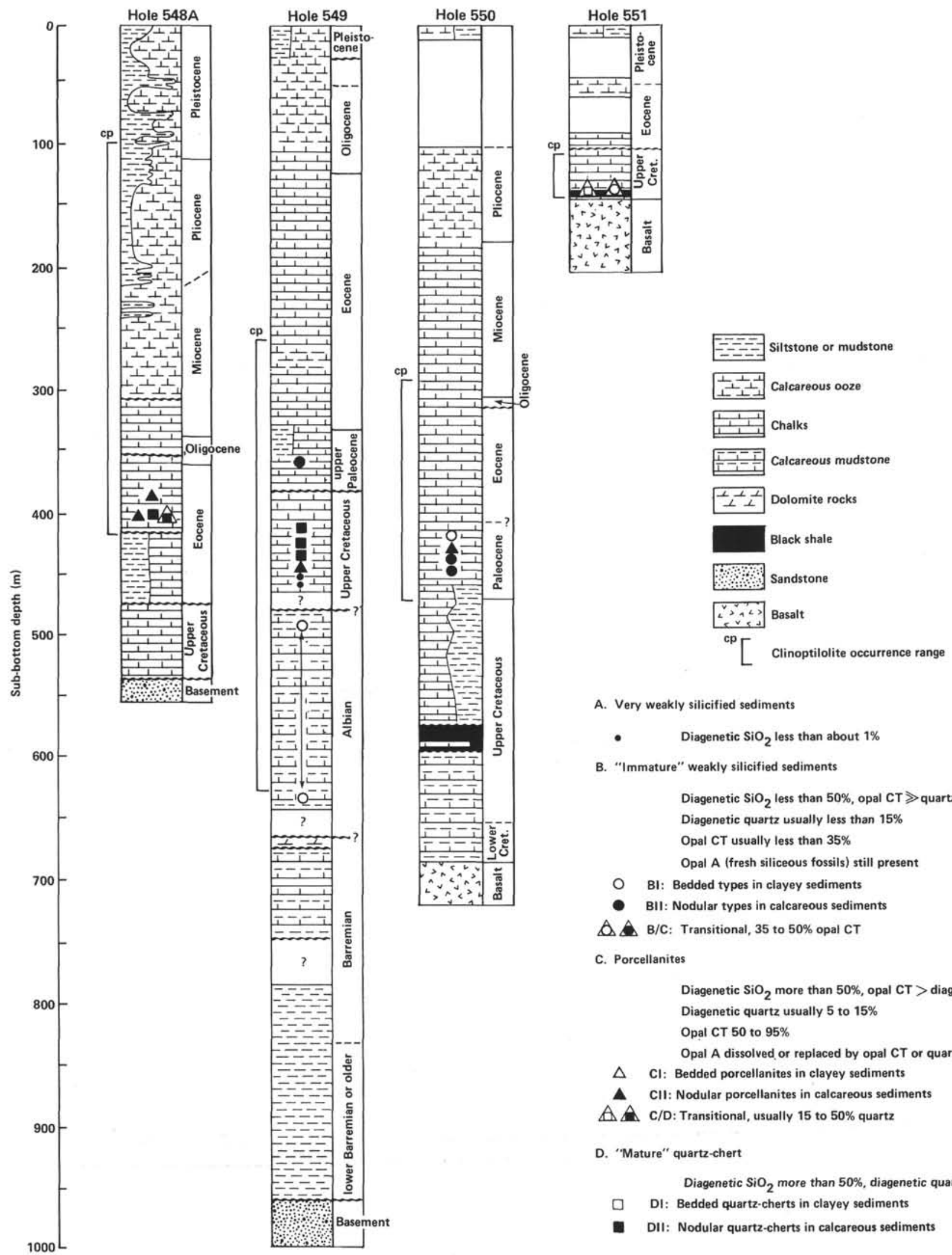

A. Very weakly silicified sediments

- Diagenetic $\mathrm{SiO}_{2}$ less than about $1 \%$

B. "Immature" weakly silicified sediments

Diagenetic $\mathrm{SiO}_{2}$ less than $50 \%$, opal $\mathrm{CT} \gg$ quartz Diagenetic quartz usually less than $15 \%$

Opal CT usually less than $35 \%$

Opal A (fresh siliceous fossils) still present

O BI: Bedded types in clayey sediments

- BII: Nodular types in calcareous sediments

A. B/C: Transitional, 35 to $50 \%$ opal CT

c. Porcellanites

Diagenetic $\mathrm{SiO}_{2}$ more than $50 \%$, opal $\mathrm{CT}>$ diagenetic quartz Diagenetic quartz usually 5 to $15 \%$

Opal CT 50 to $95 \%$

Opal A dissolved or replaced by opal CT or quartz

$\triangle \quad \mathrm{Cl}$ : Bedded porcellanites in clayey sediments

A CII: Nodular porcellanites in calcareous sediments

A C/D: Transitional, usually 15 to $50 \%$ quartz

D. "Mature" quartz-chert

Diagenetic $\mathrm{SiO}_{2}$ more than $50 \%$, diagenetic quartz $\gg$ opal CT

$\square \quad$ DI: Bedded quartz-cherts in clayey sediments

DII: Nodular quartz-cherts in calcareous sediments

Figure 1. Lithostratigraphy and distribution of silicified sediments at Sites 548 through 551. Legend shows classification of silicified sediments. 
or olive-gray (5Y 3/2) laminated, calcareous, firm siltstone with distinct bioturbated mottles. The contents of terrigenous materials such as detrital quartz and clay minerals are significant through the unit. Considerable amounts of siliceous remains (radiolarians and sponge spicules), transformed into opal CT, are scattered in a terrigenous clayey matrix.

Clinoptilolite-bearing sediments at Site 549 range from Section 549-8-2 (middle to lower Eocene, sub-bottom depth $257.7 \mathrm{~m}$ ) to Section 549-47-4 (lower Albian, subbottom depth $621.1 \mathrm{~m}$ ); that is, they appear at a shallower depth than silicified sediments, but disappear at almost the same horizon at their lower extremity.

\section{Site $\mathbf{5 5 0}$}

Siliceous biogenic remains such as radiolarians and sponge spicules are fairly abundant in some parts of the light-colored marly nannofossil chalk in the upper sediment unit, but diagenetically silicified sediments with transformed silica minerals are restricted to the interval from Section 550-35-1 (upper Paleocene, sub-bottom depth $413 \mathrm{~m}$ ) to Section 550-38-6 (lower Paleocene, subbottom depth $450 \mathrm{~m}$ ) in sediment Subunit $2 \mathrm{~b}$ and Unit 3 .

The brownish to olive-gray, marly siliceous nannofossil chalk of Subunit $2 \mathrm{~b}$ contains a distinctive silicified hard-nodule zone, greenish gray (5GY 5/1), in the interval 550-36-2, 94-109 cm. Other remarkable nodules, pinkish to yellowish gray (5Y 8/1-5YR 8/1), occur in $550-36-1,130-135 \mathrm{~cm}$ and $550-36-2,32-46 \mathrm{~cm}$, which contain abundant diagenetic dolomite crystals.

Silicified nodules also occur at 550-36-3, $144 \mathrm{~cm}$; $550-37-1,0-3 \mathrm{~cm}$ and $143 \mathrm{~cm}$; and 550-38-6, 98-106 cm, in Subunit $3 \mathrm{a}$. These nodules are generally pale brown (10YR 7/3-6/3) to brown (10YR 5/6). Some have pinkish and greenish circular features like bioturbated mottles found in adjacent sediment. Very weak haloes resulting from precipitation of diagenetically formed silica minerals also surround the nodule-bearing horizon, from Core 550-35 to Core 550-39. Clinoptilolite-bearing beds range from Core 550-22 to Core 550-41, indicating a broader zone of occurrence than diagenetic silica minerals, as observed at the previous sites.

\section{Site $\mathbf{5 5 1}$}

Silicified sediments occur in lithologic Unit 4. They are green $(5 G 7 / 2-5 G 5 / 2)$ silty or siliceous chalk bands dated as Turonian. These silicified bands include fragile porcellanite-quartz-chert micronodules of granule to sand size in the host sediments of pale green $(2.5 \mathrm{Y} 8 / 2$ 2.5GY 7/1) nannofossil chalk.

Black shales of sediment Unit 5 contain abundant euhedral clinoptilolite crystals as matrix, and large $(0.1 \mathrm{~mm})$ euhedral phillipsite cross-twinned crystals are present. Radiolarian fossils weakly transformed into diagenetic opal CT and chalcedonic quartz are also scattered in the black shale matrix, but no distinct silicified sediments were recognized. Opal-CT lepisphere precipitation is evident in foraminiferal tests in the pale gray nannofossil chalk of Unit 6, but not detectable by XRD analysis of bulk sediment. Clinoptilolite occurs more widely, from
Unit 3 (lower Maestrichtian) to Unit 6 (upper Cenomanian).

\section{MODES OF OCCURRENCE OF DIAGENETIC MINERALS}

The XRD analyses of major mineralogical compositions and occurrences of diagenetic minerals are summarized in Tables 1 through 5. Silicified-sediment types in carbonate-predominant host sediments are distinctly different from those in clayey host sediments.

\section{Diagenetic Silica Minerals in Carbonate-Predominant Sediments}

Nodular porcellanites and quartz-cherts are the typical silicified sediments in the carbonate-dominant deposits such as nannofossil chalks. Very weakly silicified chalks occur around the nodules, forming a "halo zone." Siliceous fossils in the surrounding host sediments are generally not abundant, but some of these siliceous fossils around the weakly silicified "halo zones" reveal distinct dissolution pits (Plate 1, Figs. 1 and 2). These facts may indicate that dissolution of fresh biogenic silica is the initial phase of the silica diagenesis.

The next step is precipitation of opal CT in the pore spaces of the sediment matrix and within microfossils, and biogenic opal A is transformed into opal CT (Plate 2, Figs. 1-4; Plate 3, Figs. 4 and 6). The early stage of step two is manifest as the weakly silicified halo zones surrounding silicified nodules. The halo sediments have less than $1 \%$ diagenetic silica minerals, which are chiefly opal CT (Table 5).

The third step is development of opal-CT cement. The sediments of this step are represented by weakly silicified porcellaneous nodules with patchy opal-CT matrices at Site 549 (Sample 549-18-1, 22-24 cm) and Site 550 (Sample 550-38-6, 104-106 cm) (Table 5). The diagenetic silica-mineral content is 20 to $30 \%$, most of which is opal CT.

The fourth step is replacement of foraminiferal tests by chalcedonic quartz, and precipitation of diagenetic quartz in the pore spaces of fossils and matrices (Plate 1, Fig. 5). An example of this step is in Sample 548A-21-1, $56-59 \mathrm{~cm}$, where the diagenetic silica content is $64 \%$ and the ratio of opal CT to quartz is 1.4.

The fifth and the last step in silica diagenesis is the transformation of opal CT composing fossil skeletons and pore cements to quartz. Pure quartz-chert nodules are not common in Goban Spur sediment samples. One occurrence is in Sample 549-24-3, 1-3 cm. However, the transitional facies between the fourth and the fifth steps of silica diagenesis was common in the Leg 80 samples (Plate 1, Fig. 6).

Silica diagenesis in carbonate-dominant sediments seems to develop concentrically around some nodule nuclei, and it is hard to tell whether the steps from lower to higher grades of silica diagenesis are related directly to the stratigraphic position of enclosing sediments.

\section{Dolomitic Silicified Nodules}

Dolomitic porcellanite nodules were recovered in Samples 550-36-1, 133-135 cm and 550-36-2, 41-45 cm. Do- 
Table 1. Lithofacies, chronostratigraphy, and XRD mineral analyses (\%) of selected samples from Hole 548A.

\begin{tabular}{|c|c|c|c|c|c|c|c|c|}
\hline $\begin{array}{c}\text { Sample } \\
\text { (interval in } \mathrm{cm} \text { ) }\end{array}$ & $\begin{array}{l}\text { Chrono- } \\
\text { stratigraphy }\end{array}$ & $\begin{array}{l}\text { Sub-bottom } \\
\text { depth } \\
\text { (m) }\end{array}$ & Bulk lithology & Calcite & Quartz & Opal CT & $\begin{array}{l}\text { Clinop- } \\
\text { tilolite }\end{array}$ & $\begin{array}{c}\text { Clays } \\
\text { (smectite) }\end{array}$ \\
\hline $14-2,22-24$ & lower Miocene & 330.7 & Foram. nannofossil chalk & 91 & 4 & & $<1$ & $\begin{array}{c}4 \\
(3)\end{array}$ \\
\hline $14-4,90-92$ & lower Miocene & 334.4 & Foram. nannofossil chalk & 90 & 4 & & & $\begin{array}{c}6 \\
(6)\end{array}$ \\
\hline $16-2,51-52$ & $\begin{array}{l}\text { upper-lower } \\
\text { Oligocene }\end{array}$ & 350.0 & Foram. nannofossil chalk & 95 & 1 & & 2 & $\begin{array}{l}2 \\
\text { (1) }\end{array}$ \\
\hline $17-5,85-86$ & upper Eocene & 364.4 & Foram. nannofossil chalk & 89 & 3 & & 4 & $\begin{array}{l}4 \\
(2)\end{array}$ \\
\hline $18-1,70-72$ & upper Eocene & 367.7 & Foram. nannofossil chalk & 78 & 4 & & 13 & $\begin{array}{c}5 \\
(5)\end{array}$ \\
\hline $19-1,33-35$ & mid. Eocene & 376.7 & Foram. nannofossil chalk & 78 & 10 & & 7 & $\begin{array}{l}5 \\
(2)\end{array}$ \\
\hline $19-5,50-51$ & mid. Eocene & 383.0 & Foram. nannofossil chalk & 78 & 6 & & 5 & $\begin{array}{l}11 \\
(6)\end{array}$ \\
\hline $20-2,59-60$ & mid. Eocene & 388.1 & $\begin{array}{l}\text { Weakly silicified foram. } \\
\text { nannofossil chalk }\end{array}$ & 71 & 14 & 1 & 5 & $\begin{array}{c}9 \\
(5)\end{array}$ \\
\hline $21-1,56-59$ & mid. Eocene & 396.1 & $\begin{array}{l}\text { Calcareous porcellanite } \\
\text { nodule }\end{array}$ & 24 & 28 & 37 & & $\begin{array}{l}11 \\
(11)\end{array}$ \\
\hline $21-1,56-59$ & mid. Eocene & 396.1 & $\begin{array}{l}\text { Outer crust of calcareous } \\
\text { porcellanite nodule }\end{array}$ & 78 & 11 & 11 & & \\
\hline $21-1,93-95$ & mid. Eocene & 396.5 & $\begin{array}{l}\text { Very weakly silicified } \\
\text { foram. nannofossil } \\
\text { chalk }\end{array}$ & 87 & 13 & & & \\
\hline $21-2,74-75$ & mid. Eocene & 397.8 & Foram. nannofossil chalk & 70 & 12 & & 5 & $\begin{array}{l}13 \\
(7)\end{array}$ \\
\hline $21-2,133-136$ & mid. Eocene & 398.4 & $\begin{array}{l}\text { Quartzose porcellanite } \\
\text { nodule }\end{array}$ & 5 & 28 & 29 & 12 & $\stackrel{27}{(-)}$ \\
\hline $21, \mathrm{CC}$ & mid. Eocene & 401.5 & $\begin{array}{l}\text { Outer crust of porcella- } \\
\text { neous quartz-chert } \\
\text { nodule }\end{array}$ & 84 & 16 & & & \\
\hline $21, \mathrm{CC}$ & mid. Eocene & 401.5 & $\begin{array}{l}\text { Edge of porcellaneous } \\
\text { quartz-chert nodule }\end{array}$ & 59 & 21 & 20 & & \\
\hline $21, \mathrm{CC}$ & mid. Eocene & 401.5 & $\begin{array}{l}\text { Porcellaneous quartz- } \\
\text { chert nodule }\end{array}$ & 12 & 54 & 34 & & \\
\hline $22-2,91-92$ & mid. Eocene & 407.4 & Foram. nannofossil chalk & 75 & 7 & & 4 & $\begin{array}{l}14 \\
(9)\end{array}$ \\
\hline $22-5,62-65$ & mid. Eocene & 411.6 & $\begin{array}{l}\text { Foram. marly nannofos- } \\
\text { sil chalk }\end{array}$ & 61 & 20 & & 7 & $\stackrel{12}{(-)}$ \\
\hline $22-6,106-108$ & lower Eocene & 413.6 & Marly nannofossil chalk & 31 & 33 & & & $\begin{array}{l}36 \\
(10)\end{array}$ \\
\hline $22-7,20-22$ & lower Eocene & 414.2 & Marly nannofossil chalk & 30 & 35 & & 8 & $\begin{array}{l}27 \\
\text { (11) }\end{array}$ \\
\hline $23-2,64-65$ & lower Eocene & 416.7 & $\begin{array}{l}\text { Calcareous silty mud- } \\
\text { stone }\end{array}$ & 17 & 27 & & & $\begin{array}{l}56 \\
(18)\end{array}$ \\
\hline
\end{tabular}

Note: Dashes and blanks both mean not detected by method used.

lomite occurs as euhedral rhombic crystals in a matrix of opal CT. Veins of chalcedonic quartz cut through the matrix. Siliceous fossils, mainly radiolarians, transformed into opal CT and quartz, are scattered throughout the matrix together with dolomite crystals (Plate 3, Fig. 5).

\section{Diagenetic Silica Minerals in Clayey Sediments}

Bedded silicified sediments in clayey host sediments have a restricted occurrence in Leg 80 cores. Except for local and transitional silicified sediments, middle to lower Albian gray calcareous siltstone beds from Core 549-32 to Core $549-48$ are the only major bedded silicified sediments recovered during Leg 80 .

Lithofacies are almost uniform through this siltstone sediment unit. Terrigenous materials are fairly abundant, generally as silt-size detrital quartz $(10-20 \%$ or so) and clay minerals (20-30\%). Calcareous components make up 20 to $80 \%$ of the unit, and consist mainly of nannofossils, foraminifers, and their fragments. Siliceous biogenic components, chiefly radiolarians and sponge spic- ules, are commonly scattered through a terrigenous clayey matrix.

Diagenetic silica facies are also almost uniform, particularly in the upper to middle part of these weakly silicified calcareous siltstone beds. Through the beds, siliceous fossil skeletons have generally been transformed into opal CT. Opal-CT lepispheres have also been precipitated in pore spaces of microfossils and matrix. Patchy opal-CT microlenses are scattered in some places. Euhedral clinoptilolite crystals also occur in these pore spaces. Foraminiferal tests generally remain as calcite (Plate 1, Figs. 3 and 4; Plate 2, Figs. 5 and 6). In the deeper layers of this sediment unit, from Core 549-43 to Core $549-48$, the grade of diagenesis is slightly higher, with precipitation of chalcedonic quartz having occurred sporadically in pore spaces. Patches or microlenses of chalcedonic quartz are present locally, and foraminiferal tests have been replaced by opal CT or quartz at the deeper levels (Table 5).

The content of diagenetic silica minerals ranges from less than $1 \%$ to $30 \%$ in samples from this bedded silici- 
Table 2. Lithofacies, chronostratigraphy, and XRD mineral analyses (\%) of selected samples from Hole 549.

\begin{tabular}{|c|c|c|c|c|c|c|c|c|}
\hline $\begin{array}{c}\text { Sample } \\
\text { (interval in } \mathrm{cm} \text { ) }\end{array}$ & $\begin{array}{l}\text { Chrono- } \\
\text { stratigraphy }\end{array}$ & $\begin{array}{l}\text { Sub-bottom } \\
\text { depth } \\
\text { (m) }\end{array}$ & Bulk lithology & Calcite & Quartz & Opal CT & $\begin{array}{l}\text { Clinop- } \\
\text { tilolite }\end{array}$ & $\begin{array}{c}\text { Clays } \\
\text { (smectite) }\end{array}$ \\
\hline $2-2,44-46$ & upper Eocene & 200.5 & Nannofossil chalk & 82 & 6 & & & $\begin{array}{l}12 \\
(7)\end{array}$ \\
\hline $8-2,72-73$ & mid. Eocene & 257.7 & Nannofossil chalk & 69 & 10 & & 2 & $\begin{array}{c}19 \\
(10)\end{array}$ \\
\hline $9-2,56-57$ & mid. Eocene & 267.1 & Nannofossil chalk & 74 & 5 & & 4 & $\begin{array}{l}17 \\
(8)\end{array}$ \\
\hline $10-5,51-52$ & lower Eocene & 281.0 & Marly nannofossil chalk & 72 & 7 & & 9 & $\begin{array}{l}12 \\
(9)\end{array}$ \\
\hline $11-2,59-60$ & lower Eocene & 286.1 & Marly nannofossil chalk & 15 & 26 & & 5 & $\begin{array}{l}54 \\
(22)\end{array}$ \\
\hline $14-2,55-56$ & lower Eocene & 314.6 & Marly nannofossil chalk & 63 & 5 & & 2 & $\begin{array}{l}30 \\
(20)\end{array}$ \\
\hline $15-2,56-57$ & lower Eocene & 324.1 & Calcareous claystone & 39 & 17 & & 2 & $\begin{array}{l}42 \\
(19)\end{array}$ \\
\hline $17-2,79-80$ & upper Paleocene & 343.3 & $\begin{array}{l}\text { Siliceous marly nanno- } \\
\text { fossil chalk }\end{array}$ & 53 & 7 & & 1 & $\begin{array}{l}39 \\
(34)\end{array}$ \\
\hline $18-1,22-24$ & upper Paleocene & 350.7 & $\begin{array}{l}\text { Weakly silicified calcar- } \\
\text { eous porcellanite } \\
\text { nodule }\end{array}$ & 55 & 3 & 29 & & $\begin{array}{l}13 \\
(9)\end{array}$ \\
\hline $18-2,96-99$ & upper Paleocene & 353.0 & $\begin{array}{l}\text { Siliceous nannofossil } \\
\text { chalk }\end{array}$ & 94 & 6 & & & \\
\hline $18-2,96-99$ & upper Paleocene & 353.0 & $\begin{array}{l}\text { Clayey concretion in } \\
\text { siliceous nannofos- } \\
\text { sil chalk }\end{array}$ & 33 & 2 & & & $\begin{array}{l}65 \\
(58)\end{array}$ \\
\hline $19-2,41-42$ & upper Paleocene & 361.9 & $\begin{array}{l}\text { Siliceous marly nanno- } \\
\text { fossil chalk }\end{array}$ & 63 & 5 & & & $\begin{array}{l}32 \\
(29)\end{array}$ \\
\hline $20-5,9-10$ & upper Paleocene & 375.6 & $\begin{array}{l}\text { Siliceous marly nanno- } \\
\text { fossil chalk }\end{array}$ & 57 & 8 & & 10 & $\begin{array}{c}25 \\
(19)\end{array}$ \\
\hline $21-2,19-20$ & lower Paleocene & 380.7 & Nannofossil chalk & 73 & 5 & & 6 & $\begin{array}{l}16 \\
(13)\end{array}$ \\
\hline $22-1,2-3$ & Maestrichtian & 388.5 & $\begin{array}{l}\text { Clayey concretion in } \\
\text { nannofossil chalk }\end{array}$ & 20 & 29 & & 6 & $\begin{array}{l}45 \\
\text { (7) }\end{array}$ \\
\hline $22-2,105-106$ & Maestrichtian & 391.1 & Nannofossil chalk & 95 & $<1$ & & 3 & $\begin{array}{l}2 \\
\text { (1) }\end{array}$ \\
\hline $22, \mathrm{CC}$ & Maestrichtian & 395.8 & Nannofossil chalk & 79 & 3 & & & $\begin{array}{l}18 \\
(7)\end{array}$ \\
\hline $23-2,54-55$ & Maestrichtian & 400.0 & Nannofossil chalk & 94 & $<1$ & & 3 & $\begin{array}{l}3 \\
(2)\end{array}$ \\
\hline $24-2,18-19$ & Campanian & 409.2 & Nannofossil chalk & 92 & 2 & & 3 & $\begin{array}{c}3 \\
(2)\end{array}$ \\
\hline $24-3,1-3$ & Campanian & 410.5 & Quartz-chert nodule & & $\approx 100$ & & & \\
\hline $25-2,24-25$ & $\begin{array}{l}\text { Santonian- } \\
\text { Coniacian }\end{array}$ & 418.8 & Nannofossil chalk & 86 & 5 & 2 & 3 & $\begin{array}{l}4 \\
(2)\end{array}$ \\
\hline $25-2,39-41$ & $\begin{array}{l}\text { Santonian- } \\
\text { Coniacian }\end{array}$ & 418.9 & Nannofossil chalk & 93 & 7 & & & \\
\hline $25-2,53-55$ & $\begin{array}{l}\text { Santonian- } \\
\text { Coniacian }\end{array}$ & 419.0 & $\begin{array}{l}\text { Calcareous porcella- } \\
\text { neous quartz-chert }\end{array}$ & 11 & 50 & 39 & & \\
\hline $26-1,8-9$ & Turonian & 426.6 & $\begin{array}{l}\text { Porcellaneous quartz- } \\
\text { chert }\end{array}$ & 6 & 60 & 34 & & \\
\hline $26-1,24-25$ & Turonian & 426.8 & Nannofossil chalk & 84 & 5 & 1 & 4 & $\begin{array}{l}6 \\
(4)\end{array}$ \\
\hline $26-1,46-48$ & Turonian & 427.0 & Nannofossil chalk & 81 & 5 & & 5 & $\begin{array}{c}9 \\
(3)\end{array}$ \\
\hline $27-1,29-30$ & $\begin{array}{l}\text { Turonian- } \\
\text { Cenomanian }\end{array}$ & 436.3 & $\begin{array}{l}\text { Calcareous porcellanite } \\
\text { in black shale }\end{array}$ & 31 & 15 & 49 & 5 & \\
\hline $27-1,42-43$ & $\begin{array}{l}\text { Turonian- } \\
\text { Cenomanian }\end{array}$ & 436.4 & $\begin{array}{l}\text { Laminated black } \\
\text { carbonaceous shale }\end{array}$ & 3 & 26 & & 27 & $\begin{array}{c}44 \\
(32)\end{array}$ \\
\hline $28-1,73-74$ & Cenomanian & 446.2 & Nannofossil chalk & 69 & 12 & & 9 & $\begin{array}{l}10 \\
(5)\end{array}$ \\
\hline $28-2,25-26$ & Cenomanian & 447.3 & Nannofossil chalk & 75 & 9 & 2 & 8 & $\begin{array}{l}6 \\
(4)\end{array}$ \\
\hline $28-2,30-32$ & Cenomanian & 447.3 & $\begin{array}{l}\text { Foram. nannofossil } \\
\text { chalk }\end{array}$ & 72 & 12 & & 10 & $\begin{array}{l}6 \\
(6)\end{array}$ \\
\hline $29-1,19-20$ & Cenomanian & 455.2 & Nannofossil chalk & 67 & 15 & 7 & 5 & $\begin{array}{l}6 \\
(4)\end{array}$ \\
\hline $32-1,17-18$ & mid. Albian & 483.7 & $\begin{array}{l}\text { Gray calcareous silt- } \\
\text { stone }\end{array}$ & 22 & 27 & 25 & 6 & $\begin{array}{l}20 \\
(6)\end{array}$ \\
\hline $32-1,35-37$ & mid. Albian & 483.9 & $\begin{array}{l}\text { Gray calcareous silt- } \\
\text { stone }\end{array}$ & 34 & 19 & 20 & 7 & $\stackrel{20}{(-)}$ \\
\hline $32, \mathrm{CC}$ & mid. Albian & 484.1 & $\begin{array}{l}\text { Gray calcareous silt- } \\
\text { stone }\end{array}$ & 46 & 22 & 11 & 7 & $\begin{array}{l}14 \\
(5)\end{array}$ \\
\hline $34-1,52-53$ & mid. Albian & 503.0 & $\begin{array}{l}\text { Gray calcareous silt- } \\
\text { stone }\end{array}$ & 51 & 19 & 16 & 4 & $\begin{array}{l}10 \\
(2)\end{array}$ \\
\hline $34-1,55-56$ & mid. Albian & 503.1 & $\begin{array}{l}\text { Gray calcareous silt- } \\
\text { stone }\end{array}$ & 63 & 15 & 7 & 8 & $\stackrel{7}{(-)}$ \\
\hline
\end{tabular}


Table 2. (Continued).

\begin{tabular}{|c|c|c|c|c|c|c|c|c|}
\hline $\begin{array}{c}\text { Sample } \\
\text { (interval in } \mathrm{cm} \text { ) }\end{array}$ & $\begin{array}{l}\text { Chrono- } \\
\text { stratigraphy }\end{array}$ & $\begin{array}{l}\text { Sub-bottom } \\
\text { depth } \\
\text { (m) }\end{array}$ & Bulk lithology & Calcite & Quartz & Opal CT & $\begin{array}{l}\text { Clinop- } \\
\text { tilolite }\end{array}$ & $\begin{array}{c}\text { Clays } \\
\text { (smectite) }\end{array}$ \\
\hline $35-1,37-39$ & mid. Albian & 512.4 & $\begin{array}{l}\text { Gray calcareous silt- } \\
\text { stone }\end{array}$ & 35 & 21 & 30 & 7 & $\stackrel{7}{(-)}$ \\
\hline $35-1,139-141$ & mid. Albian & 513.4 & $\begin{array}{l}\text { Gray calcareous silt- } \\
\text { stone }\end{array}$ & 26 & 23 & 20 & 13 & $\begin{array}{l}18 \\
(9)\end{array}$ \\
\hline $35, \mathrm{CC}(2-3)$ & mid. Albian & 513.7 & $\begin{array}{l}\text { Gray calcareous silt- } \\
\text { stone }\end{array}$ & 66 & 10 & & 5 & $\begin{array}{l}19 \\
(6)\end{array}$ \\
\hline $36-1,5-7$ & Albian & 521.6 & $\begin{array}{l}\text { Gray calcareous silt- } \\
\text { stone }\end{array}$ & 73 & 12 & 9 & 6 & \\
\hline $36-1,15-17$ & Albian & 521.7 & $\begin{array}{l}\text { Laminated calcareous } \\
\text { sandy siltstone }\end{array}$ & 36 & 26 & & 21 & $\stackrel{17}{(-)}$ \\
\hline $36-1,20-21$ & Albian & 521.7 & $\begin{array}{l}\text { Gray calcareous silt- } \\
\text { stone }\end{array}$ & 49 & 25 & 6 & 5 & $\begin{array}{l}15 \\
(5)\end{array}$ \\
\hline $37-1,20-21$ & Albian & 522.7 & $\begin{array}{l}\text { Gray calcareous silt- } \\
\text { stone }\end{array}$ & 19 & 18 & 8 & 15 & $\begin{array}{l}39 \\
(8)\end{array}$ \\
\hline $37-1,31-32$ & Albian & 522.8 & $\begin{array}{l}\text { Gray calcareous silt- } \\
\text { stone }\end{array}$ & 23 & 19 & & 21 & $\begin{array}{l}37 \\
(15)\end{array}$ \\
\hline $37-2,36-38$ & Albian & 524.4 & $\begin{array}{l}\text { Gray calcareous silt- } \\
\text { stone }\end{array}$ & 43 & 12 & & 11 & $\begin{array}{l}34 \\
(8)\end{array}$ \\
\hline $38-1,34-35$ & Albian & 531.4 & Nannofossil chalk & 83 & 5 & 3 & 2 & $\begin{array}{l}7 \\
\text { (2) }\end{array}$ \\
\hline $39-1,6-7$ & Albian & 540.6 & $\begin{array}{l}\text { Gray calcareous silt- } \\
\text { stone }\end{array}$ & 32 & 22 & 9 & 9 & $\begin{array}{l}28 \\
(9)\end{array}$ \\
\hline $40-1,7-8$ & Albian & 550.1 & $\begin{array}{l}\text { Gray calcareous silt- } \\
\text { stone }\end{array}$ & 33 & 21 & 9 & 8 & $\begin{array}{l}29 \\
\text { (9) }\end{array}$ \\
\hline $42-1,38-39$ & Albian & 569.4 & $\begin{array}{l}\text { Light gray calcareous } \\
\text { siltstone }\end{array}$ & 72 & 9 & 1 & & $\begin{array}{l}18 \\
(5)\end{array}$ \\
\hline $42-2,36-37$ & Albian & 570.9 & $\begin{array}{l}\text { Gray calcareous silt- } \\
\text { stone }\end{array}$ & 41 & 21 & 11 & 6 & $\begin{array}{l}21 \\
(5)\end{array}$ \\
\hline $42-2,38-40$ & Albian & 570.9 & $\begin{array}{l}\text { Gray calcareous silt- } \\
\text { stone }\end{array}$ & 44 & 11 & 8 & 10 & $\begin{array}{l}27 \\
(7)\end{array}$ \\
\hline $43-2,88-90$ & Albian & 580.9 & $\begin{array}{l}\text { Gray calcareous silt- } \\
\text { stone }\end{array}$ & 28 & 32 & 7 & 3 & $\begin{array}{l}30 \\
(10)\end{array}$ \\
\hline $43-3,127-130$ & Albian & 528.8 & $\begin{array}{l}\text { Gray calcareous silt- } \\
\text { stone }\end{array}$ & 30 & 16 & 8 & 10 & $\begin{array}{l}36 \\
(8)\end{array}$ \\
\hline $44-2,110-111$ & Albian & 590.6 & $\begin{array}{l}\text { Gray calcareous silt- } \\
\text { stone }\end{array}$ & 58 & 15 & 11 & 2 & $\begin{array}{l}14 \\
(6)\end{array}$ \\
\hline $44-3,141-143$ & Albian & 592.4 & $\begin{array}{l}\text { Gray calcareous silt- } \\
\text { stone }\end{array}$ & 37 & 18 & & 10 & $\begin{array}{l}35 \\
(9)\end{array}$ \\
\hline $45-3,40-41$ & Albian & 600.9 & $\begin{array}{l}\text { Gray calcareous silt- } \\
\text { stone }\end{array}$ & 40 & 24 & 16 & 3 & $\begin{array}{l}17 \\
(7)\end{array}$ \\
\hline $45-3,130-133$ & Albian & 601.8 & $\begin{array}{l}\text { Gray calcareous silt- } \\
\text { stone }\end{array}$ & 35 & 15 & 6 & 7 & $\begin{array}{l}37 \\
(7)\end{array}$ \\
\hline $46-3,31-33$ & Albian & 610.3 & $\begin{array}{l}\text { Gray calcareous silt- } \\
\text { stone }\end{array}$ & 56 & 14 & 6 & 7 & $\stackrel{17}{(-)}$ \\
\hline $46-4,91-92$ & Albian & 612.4 & $\begin{array}{l}\text { Gray calcareous silt- } \\
\text { stone }\end{array}$ & 32 & 20 & 11 & 7 & $\begin{array}{l}30 \\
(10)\end{array}$ \\
\hline $47-1,46-47$ & Albian & 617.0 & $\begin{array}{l}\text { Gray calcareous silt- } \\
\text { stone }\end{array}$ & 32 & 22 & 10 & 5 & $\begin{array}{l}31 \\
(8)\end{array}$ \\
\hline $47-2,92-94$ & Albian & 618.9 & $\begin{array}{l}\text { Gray calcareous silt- } \\
\text { stone }\end{array}$ & 47 & 17 & & & $\begin{array}{l}36 \\
(9)\end{array}$ \\
\hline $47-3,84-86$ & Albian & 620.4 & $\begin{array}{l}\text { Gray calcareous silt- } \\
\text { stone }\end{array}$ & 46 & 24 & 8 & & $\begin{array}{l}22 \\
(9)\end{array}$ \\
\hline $47-4,14-17$ & Albian & 621.2 & $\begin{array}{l}\text { Gray calcareous silt- } \\
\text { stone }\end{array}$ & 39 & 27 & 4 & 7 & $\begin{array}{l}22 \\
(8)\end{array}$ \\
\hline $47-4,24-26$ & Albian & 621.3 & $\begin{array}{l}\text { Gray calcareous silt- } \\
\text { stone }\end{array}$ & 71 & 9 & & & $\begin{array}{l}20 \\
(7)\end{array}$ \\
\hline $47-4,72-73$ & Albian & 621.7 & $\begin{array}{l}\text { Gray calcareous silt- } \\
\text { stone }\end{array}$ & 40 & 32 & & & $\begin{array}{l}28 \\
(11)\end{array}$ \\
\hline $48-1,33-34$ & Albian & 626.3 & $\begin{array}{l}\text { Gray calcareous silt- } \\
\text { stone }\end{array}$ & 49 & 24 & 5 & & $\begin{array}{r}22 \\
5\end{array}$ \\
\hline
\end{tabular}

Note: Dashes and blanks both mean not detected by method used.

fied siltstone. Almost all of the diagenetic silica is opal $\mathrm{CT}$, but diagenetic quartz appears in the deeper parts of the unit. These diagenetic facies correspond to the second to fourth steps of silica diagenesis in the case of carbonate-predominant sediments, previously outlined. But development of opal-CT cement, the third step, is poor in these clayey sediments. Slight but detectable progression of diagenetic silica facies with increase in subbottom depth is a characteristic feature in the clayey sediments.

\section{OPAL-CT LATTICE SPACING}

The $d(101)$ spacing of opal CT is considered a good index of the degree of silica diagenesis. Murata and Larson (1975) recognized a decrease in the $d(101)$ spacing of opal CT with burial depth in the Monterey Shale, and attributed this to a progressive diagenetic ordering. Such a decrease or ordering of $d$ spacing of opal CT with burial depth has been reported by some other investigators (von Rad et al., 1978; Iijima et al., 1980). 
Table 3. Lithofacies, chronostratigraphy, and XRD mineral analyses (\%) of selected samples from Hole 550.

\begin{tabular}{|c|c|c|c|c|c|c|c|c|c|c|}
\hline $\begin{array}{c}\text { Sample } \\
\text { (interval in } \mathrm{cm} \text { ) }\end{array}$ & $\begin{array}{l}\text { Chrono- } \\
\text { stratigraphy }\end{array}$ & $\begin{array}{l}\text { Sub-bottom } \\
\text { depth } \\
\text { (m) }\end{array}$ & Bulk lithology & Calcite & Dolomite & Quartz & Opal CT & Opal A & $\begin{array}{l}\text { Clinop- } \\
\text { tilolite }\end{array}$ & $\begin{array}{c}\text { Clays } \\
\text { (smectite) }\end{array}$ \\
\hline $21-3,75$ & lower Miocene & 283.7 & Marly nannofossil chalk & 79 & & 7 & & & & $\stackrel{14}{(-)}$ \\
\hline $22-2,18-19$ & lower Miocene & 291.2 & Marly nannofossil chalk & 58 & & 11 & & & 2 & $\begin{array}{l}29 \\
(12)\end{array}$ \\
\hline $23-2,47-48$ & lower miocene & 301.0 & $\begin{array}{l}\text { Marly nannofossil chalk } \\
\text { turbidite layer }\end{array}$ & 58 & & 11 & & 7 & 2 & $\begin{array}{l}22 \\
\text { (11) }\end{array}$ \\
\hline $24-4,104$ & lower Eocene & 314.0 & Claystone & 6 & & 14 & & & 6 & $\begin{array}{c}73 \\
(21)\end{array}$ \\
\hline $24-4,108$ & lower Eocene & 314.1 & Calcareous claystone & 24 & & 18 & & & & $\begin{array}{l}58 \\
(27)\end{array}$ \\
\hline $25-1,38-39$ & lower Eocene & 318.4 & Marly nannofossil chalk & 49 & & 13 & & & 5 & $\begin{array}{l}33 \\
(15)\end{array}$ \\
\hline $28-1,46-47$ & lower Eocene & 347.0 & Marly nannofossil chalk & 58 & & 10 & & & & $\begin{array}{l}32 \\
(18)\end{array}$ \\
\hline $28-1,68-69$ & lower Eocene & 347.2 & Marly nannofossil chalk & 65 & & 7 & & & 4 & $\begin{array}{l}24 \\
(14)\end{array}$ \\
\hline $29-2,68-69$ & lower Eocene & 358.2 & Marly nannofossil chalk & 76 & & 4 & & & $<1$ & $\begin{array}{l}20 \\
(11)\end{array}$ \\
\hline $34-4,118$ & upper Paleocene & 409.2 & $\begin{array}{l}\text { Siliceous marly nanno- } \\
\text { fossil chalk }\end{array}$ & 65 & & 13 & & & & $\begin{array}{l}22 \\
(12)\end{array}$ \\
\hline $34-5,118$ & upper Paleocene & 410.7 & Calcareous claystone & 41 & & 13 & & & & $\begin{array}{l}46 \\
(31)\end{array}$ \\
\hline $34-5,119$ & upper Paleocene & 410.7 & $\begin{array}{l}\text { Calcareous sandy } \\
\text { claystone }\end{array}$ & 46 & & 19 & & & 9 & $\begin{array}{l}26 \\
(18)\end{array}$ \\
\hline $34-5,145$ & upper Paleocene & 410.9 & Claystone & & & 13 & & & 10 & $\begin{array}{l}77 \\
(60)\end{array}$ \\
\hline $35-1,23-24$ & upper Paleocene & 413.2 & $\begin{array}{l}\text { Weakly silicified calcar- } \\
\text { eous mudstone }\end{array}$ & 57 & & 3 & 13 & & 6 & $\begin{array}{l}21 \\
(21)\end{array}$ \\
\hline $35-1,136$ & upper Paleocene & 414.4 & Calcareous mudstone & 50 & & 12 & & & & $\begin{array}{c}38 \\
(38)\end{array}$ \\
\hline $35-2,12-13$ & upper Paleocene & 414.6 & Claystone & 10 & & 5 & & & & $\begin{array}{l}85 \\
\text { (73) }\end{array}$ \\
\hline $35-5,12-13$ & upper Paleocene & 419.1 & Claystone & 3 & & 12 & & & & $\begin{array}{l}85 \\
(70)\end{array}$ \\
\hline $35-5,69-71$ & upper Paleocene & 419.7 & $\begin{array}{l}\text { Nannofossil-bearing } \\
\text { siliceous claystone }\end{array}$ & 25 & & 11 & & & & $\begin{array}{l}64 \\
(54)\end{array}$ \\
\hline $35-5,87-89$ & upper Paleocene & 419.9 & $\begin{array}{l}\text { White foram. marly } \\
\text { nannofossil chalk }\end{array}$ & 57 & & 7 & & & & $\begin{array}{c}36 \\
(20)\end{array}$ \\
\hline $35-5,87-89$ & upper Paleocene & 419.9 & $\begin{array}{l}\text { Brown siliceous clay- } \\
\text { stone }\end{array}$ & 30 & & 15 & & & & $\begin{array}{l}55 \\
(39)\end{array}$ \\
\hline $35-5,105$ & upper Paleocene & 421.0 & Calcareous claystone & 42 & & 14 & & & 6 & $\begin{array}{l}39 \\
(31)\end{array}$ \\
\hline $35-5,138$ & upper Paleocene & 421.3 & $\begin{array}{l}\text { Green to pinkish streak } \\
\text { of claystone }\end{array}$ & 11 & & 2 & & & & $\begin{array}{l}87 \\
(84)\end{array}$ \\
\hline $36-1,12-15$ & upper Paleocene & 422.6 & $\begin{array}{l}\text { Laminated calcareous } \\
\text { mudstone }\end{array}$ & 47 & & 4 & & & 10 & $\begin{array}{l}38 \\
(38)\end{array}$ \\
\hline $36-1,13-15$ & upper Paleocene & 422.6 & Calcareous claystone & 13 & & 18 & & & & $\begin{array}{l}69 \\
(69)\end{array}$ \\
\hline $36-1,132-135$ & upper Paleocene & 423.8 & $\begin{array}{l}\text { Porcellaneous dolomite } \\
\text { nodule }\end{array}$ & 7 & 68 & 5 & 20 & & & \\
\hline $36-1,134$ & upper Paleocene & 423.8 & $\begin{array}{l}\text { Outer part of porcella- } \\
\text { neous (dolomite) } \\
\text { nodule }\end{array}$ & 22 & & 5 & 57 & & & $\begin{array}{l}16 \\
(16)\end{array}$ \\
\hline $36-2,41-45$ & upper Paleocene & 424.4 & $\begin{array}{l}\text { Dolomitic porcellanite } \\
\text { nodule }\end{array}$ & & 12 & 8 & 67 & & & $\begin{array}{c}13 \\
(13)\end{array}$ \\
\hline $36-2,41-45$ & upper Paleocene & 424.4 & $\begin{array}{l}\text { Outer crust of dolomit- } \\
\text { ic porcellanite } \\
\text { nodule }\end{array}$ & 5 & & 3 & 18 & & & $\begin{array}{l}73 \\
(73)\end{array}$ \\
\hline $36-2,134$ & upper Paleocene & 425.3 & Claystone & 11 & & 31 & & & 30 & $\begin{array}{c}28 \\
(17)\end{array}$ \\
\hline $36-3,126$ & upper Paleocene & 426.8 & $\begin{array}{l}\text { Pink mottle in marly } \\
\text { nannofossil chalk }\end{array}$ & 39 & & 2 & & & & $\begin{array}{l}59 \\
(39)\end{array}$ \\
\hline $37-1,0-3$ & upper Paleocene & 432.0 & $\begin{array}{l}\text { Brownish calcareous } \\
\text { porcellanite nodule }\end{array}$ & 23 & & 17 & 60 & & & \\
\hline $37-1,0-3$ & upper Paleocene & 432.0 & $\begin{array}{l}\text { White part of calcare- } \\
\text { ous porcellanite } \\
\text { nodule }\end{array}$ & 81 & & 6 & 13 & & & \\
\hline $37-1,143$ & upper Paleocene & 433.4 & $\begin{array}{l}\text { Green-pink siliceous } \\
\text { concretion in } \\
\text { nannofossil chalk }\end{array}$ & 32 & & 5 & 19 & & & $\begin{array}{l}44 \\
(44)\end{array}$ \\
\hline $37-2,31-32$ & upper Paleocene & 433.8 & Marly nannofossil chalk & 53 & & 3 & 3 & & 7 & $\begin{array}{l}34 \\
(34)\end{array}$ \\
\hline $37-2,140$ & upper Paleocene & 434.9 & $\begin{array}{l}\text { Green clayey concretion } \\
\text { in marly nannofos- } \\
\text { sil chalk }\end{array}$ & 14 & & & & & & $\begin{array}{l}86 \\
(75)\end{array}$ \\
\hline
\end{tabular}


Table 3. (Continued).

\begin{tabular}{|c|c|c|c|c|c|c|c|c|c|c|}
\hline $\begin{array}{c}\text { Sample } \\
\text { (interval in cm) }\end{array}$ & $\begin{array}{l}\text { Chrono- } \\
\text { stratigraphy }\end{array}$ & $\begin{array}{l}\text { Sub-bottom } \\
\text { depth } \\
\text { (m) }\end{array}$ & Bulk lithology & Calcite & Dolomite & Quartz & Opal CT & Opal A & $\begin{array}{l}\text { Clinop- } \\
\text { tilolite }\end{array}$ & $\begin{array}{c}\text { Clays } \\
\text { (smectite) }\end{array}$ \\
\hline $37-4,31-32$ & upper Paleocene & 436.8 & Marly nannofossil chalk & 69 & & 3 & 5 & & & $\begin{array}{c}23 \\
(21)\end{array}$ \\
\hline $38-6,70$ & lower Paleocene & 449.7 & $\begin{array}{l}\text { White siliceous fossil- } \\
\text { bearing marly } \\
\text { nannofossil chalk }\end{array}$ & 64 & & 5 & & & 16 & $\begin{array}{c}15 \\
(12)\end{array}$ \\
\hline $38-6,102$ & lower Paleocene & 450.0 & $\begin{array}{l}\text { Brown concretion of } \\
\text { weakly silicified } \\
\text { siliceous marly } \\
\text { nannofossil chalk }\end{array}$ & 55 & & 9 & 14 & & 6 & $\begin{array}{l}16 \\
(13)\end{array}$ \\
\hline $38-6,104-106$ & lower Paleocene & 450.1 & $\begin{array}{l}\text { White concretion of } \\
\text { weakly silicified } \\
\text { siliceous marly } \\
\text { nannofossil chalk }\end{array}$ & 44 & & 9 & 23 & & 13 & $\stackrel{11}{(-)}$ \\
\hline $38-6,104-106$ & lower Paleocene & 450.1 & $\begin{array}{l}\text { Brown concretion of } \\
\text { weakly silicified } \\
\text { siliceous marly } \\
\text { nannofossil chalk }\end{array}$ & 64 & & 5 & 12 & & 11 & $\stackrel{8}{(-)}$ \\
\hline $39-2,35-37$ & lower Paleocene & 452.9 & $\begin{array}{l}\text { Marly siliceous nanno- } \\
\text { fossil chalk }\end{array}$ & 67 & & 5 & & & 17 & $\begin{array}{l}11 \\
(4)\end{array}$ \\
\hline $39-5,92-93$ & lower Paleocene & 457.9 & Nannofossil chalk & 92 & & 2 & & & $<1$ & $\begin{array}{c}5 \\
(3)\end{array}$ \\
\hline $41-2,55-56$ & $\begin{array}{l}\text { upper } \\
\text { Maestrichtian }\end{array}$ & 472.1 & Marly nannofossil chalk & 78 & & 2 & & & 9 & $\begin{array}{l}11 \\
(9)\end{array}$ \\
\hline $42-1,9-10$ & $\begin{array}{l}\text { upper } \\
\text { Maestrichtian }\end{array}$ & 479.1 & $\begin{array}{l}\text { Sandy calcareous } \\
\text { turbidite }\end{array}$ & 45 & & 11 & & & & $\begin{array}{l}44 \\
(21)\end{array}$ \\
\hline
\end{tabular}

Note: Dashes and blanks both mean not detected by method used.

Figure 2 shows the changes of $d$ spacing of opal CT with sub-bottom depth for silicified sediment samples from all sites drilled during Leg 80 . The range of the $d$ (101) spacing of opal CT is from 4.115 to $4.069 \AA$, and the decrease or ordering of $d$ spacing with sub-bottom depth is shown by two different trends of clusters, although a few exceptions occur. The two clusters correspond to the nodular and bedded types of silica diagenesis, respectively. The $d$ spacing decreases rapidly at shallower horizons (from 395 to $450 \mathrm{~m}$ ) in nodular-type silicified sediments than in bedded silicified sediments. The $d$ spacing decreases more gradually at deeper horizons (from 480 to $620 \mathrm{~m}$ ) in the bedded silicified sediments of Site 549 .

These trends illustrate the difference in silica diagenesis between nodular and bedded types. The $d$ spacings fluctuate considerably, and do not show simple linear trends with burial depth. Thus, the overall trend is not always obvious if a limited number of samples are examined separately, particularly in the case of sporadic nodular-type silicified sediments. However, the trend of decreasing $d$ spacings for the nodular type may also indicate that unconformities do not severely perturb the relative burial depths at Sites 548, 549, and 550 since formation of the silicified nodules. Silicified sediments do occur at shallower depths at Site 551 than at the other sites, judging from the diagenetic silica facies.

Another noteworthy fact is that the spacings of opal $\mathrm{CT}$ in mature quartzose chert nodules do not fall in a narrow-spacing group. Rather, opal CT in quartzose chert nodules has wide $d$ spacings. This may indicate that quartz precipitation or mature quartz-chert formation is a different phenomenon from opal-CT ordering, and that the relation between these two silica minerals is not defined by a simple replacement of opal CT by quartz after the opal-CT lattice becomes ordered.

\section{ZEOLITES}

\section{Clinoptilolite}

X-ray diffraction analyses show occurrences of clinoptilolite over a very wide range of age, depth, and hostsediment type (Fig. 1, Tables 1-5). Clinoptilolite-bearing sediments from Leg 80 sites are hemipelagic to pelagic chalks, calcareous siltstones, calcareous sandy claystones, and black zeolitic shales. Nearly all of these sediments are devoid of tuff and volcanic glass shards, except for the black shales of Site 551 (Section 551-5-2). Stratigraphically, clinoptilolite occurs from shallower to lower levels than the zones of diagenetically silicified sediments. These facts may indicate some relationship between clinoptilolite and silica diagenesis; they also suggest that clinoptilolite originates as a precipitate from a biogenic silica solution. Clinoptilolite occurs generally as distinct euhedral crystals or aggregates of crystals in pore spaces. In the black zeolitic shales of Section 551-5-2, clinoptilolite constitutes 20 to $35 \%$ of the rock as fine matrix in both the black and white laminae, accompanied by sporadic euhedral cross-twinned phillipsite and radiolarians transformed into opal $\mathrm{CT}$ and quartz (Plate 3, Figs. 1 and 2).

\section{Phillipsite}

Phillipsite occurs only in the black shales of Section 551-5-2, as sporadic large (30-100 $\mu \mathrm{m})$ euhedral crosstwinned crystals in a fine matrix of clinoptilolite and clay minerals (Tables 4 and 5). Riech and von Rad (1979a) noted that phillipsite commonly occurs as an alteration 
Table 4. Lithofacies, chronostratigraphy, and XRD mineral analyses (\%) of selected samples from Hole 551.

\begin{tabular}{|c|c|c|c|c|c|c|c|c|c|}
\hline $\begin{array}{c}\text { Sample } \\
\text { (interval in } \mathrm{cm} \text { ) }\end{array}$ & $\begin{array}{l}\text { Chrono- } \\
\text { stratigraphy }\end{array}$ & $\begin{array}{l}\text { Sub-bottom } \\
\text { depth } \\
\text { (m) }\end{array}$ & Bulk lithology & Calcite & Quartz & Opal CT & $\begin{array}{l}\text { Clinop- } \\
\text { tilolite }\end{array}$ & $\begin{array}{c}\text { Clays } \\
\text { (smectite) }\end{array}$ & $\begin{array}{l}\text { Phillip- } \\
\text { site }\end{array}$ \\
\hline $2-1,2$ & Maestrichtian & 104.0 & $\begin{array}{l}\text { Foram. nannofossil } \\
\text { ooze }\end{array}$ & 92 & 8 & & & & \\
\hline $2-2,135-136$ & Maestrichtian & 106.9 & Calcareous clay & 89 & & & 3 & $\begin{array}{l}8 \\
(6)\end{array}$ & \\
\hline $3-2,96-97$ & lower Maestrichtian & 116.0 & Marly nannofossil ooze & 90 & 1 & & 2 & $\begin{array}{l}6 \\
(5)\end{array}$ & \\
\hline $3-3,139$ & lower Maestrichtian & 117.9 & Marly nannofossil ooze & 88 & 4 & & & $\begin{array}{r}7 \\
(-)\end{array}$ & \\
\hline $4-2,54$ & upper Campanian & 125.5 & Nannofossil chalk & 95 & 5 & & & & \\
\hline $4-2,119-120$ & upper Campanian & 126.2 & Nannofossil chalk & 87 & 1 & & 7 & $\begin{array}{c}5 \\
(3)\end{array}$ & \\
\hline $5-1,14$ & lower Turonian & 132.6 & $\begin{array}{l}\text { Nannofossil chalk with } \\
\text { white mottles }\end{array}$ & 76 & 6 & & 6 & $\begin{array}{l}12 \\
(5)\end{array}$ & \\
\hline $5-1,30$ & lower Turonian & 132.8 & Olive clay band & & & & & $\begin{array}{l}100 \\
(75)\end{array}$ & \\
\hline $5-1,48-49$ & lower Turonian & 133.0 & $\begin{array}{l}\text { Laminated calcareous } \\
\text { mudstone }\end{array}$ & 36 & 9 & & & $\begin{array}{l}55 \\
(55)\end{array}$ & \\
\hline $5-1,53-54$ & lower Turonian & 133.0 & $\begin{array}{l}\text { Laminated nannofossil } \\
\text { chalk }\end{array}$ & 64 & 8 & & 5 & $\begin{array}{l}23 \\
(13)\end{array}$ & \\
\hline $5-1,114$ & lower Turonian & 133.6 & $\begin{array}{l}\text { Laminated nannofossil } \\
\text { chalk }\end{array}$ & 73 & 5 & & 7 & $\begin{array}{l}15 \\
(5)\end{array}$ & \\
\hline $5-2,3-5$ & lower Turonian & 134.0 & $\begin{array}{l}\text { Marly silicified nanno- } \\
\text { fossil chalk with } \\
\text { porcellaneous chert } \\
\text { patches }\end{array}$ & 40 & 40 & 20 & & & \\
\hline $5-2,6$ & lower Turonian & 134.0 & $\begin{array}{l}\text { Marly silicified nanno- } \\
\text { fossil chalk with } \\
\text { porcellaneous chert } \\
\text { patches }\end{array}$ & 42 & 38 & 16 & 3 & & \\
\hline $5-2,20$ & lower Turonian & 134.2 & Nannofossil chalk & 82 & 4 & & 5 & $\begin{array}{l}9 \\
(6)\end{array}$ & \\
\hline $5-2,49-51$ & lower Turonian & 134.5 & $\begin{array}{l}\text { Marly silicified nanno- } \\
\text { fossil chalk with } \\
\text { porcellaneous chert } \\
\text { patches }\end{array}$ & 4 & 36 & 43 & 11 & $\begin{array}{c}5 \\
(5)\end{array}$ & \\
\hline $5-2,50-53$ & lower Turonian & 134.5 & $\begin{array}{l}\text { Marly silicified nanno- } \\
\text { fossil chalk with } \\
\text { porcellaneous chert } \\
\text { patches }\end{array}$ & 35 & 32 & 25 & & $\stackrel{8}{(-)}$ & \\
\hline $5-2,56$ & lower Turonian & 134.6 & Marly nannofossil chalk & 42 & 15 & & 25 & $\stackrel{17}{(\rightarrow)}$ & \\
\hline $5-2,57-58$ & lower Turonian & 134.6 & $\begin{array}{l}\text { Green and black } \\
\text { granular porcella- } \\
\text { neous cherty mud }\end{array}$ & 29 & 23 & 30 & 17 & - & \\
\hline $5-2,70$ & lower Turonian & 134.7 & $\begin{array}{l}\text { Black carbonaceous } \\
\text { zeolitic clay }\end{array}$ & - & 18 & & 37 & $\stackrel{45}{(-)}$ & \\
\hline $5-2,79-81$ & lower Turonian & 134.8 & $\begin{array}{l}\text { Black zeolitic carbona- } \\
\text { ceous mudstone } \\
\text { with zeolitic clay } \\
\text { lamina }\end{array}$ & 6 & 12 & & 29 & $\begin{array}{l}17 \\
(6)\end{array}$ & 35 \\
\hline $5-2,91-92$ & lower Turonian & 134.9 & $\begin{array}{l}\text { Marly chalk in black } \\
\text { carbonaceous } \\
\text { zeolitic clay }\end{array}$ & 44 & 13 & & 26 & $\begin{array}{l}17 \\
(6)\end{array}$ & \\
\hline $5-2,101-103$ & lower Turonian & 135.0 & $\begin{array}{l}\text { Black zeolitic carbona- } \\
\text { ceous mudstone, } \\
\text { zeolitic clay lamina }\end{array}$ & 9 & 12 & & 22 & $\stackrel{14}{(-)}$ & 40 \\
\hline $5, \mathrm{CC}$ & lower Turonian & 135.3 & $\begin{array}{l}\text { Black carbonaceous } \\
\text { zeolitic clay }\end{array}$ & - & 16 & & 34 & $\stackrel{43}{(-)}$ & 6 \\
\hline $6-1,13-14$ & upper Cenomanian & 142.2 & $\begin{array}{l}\text { Foram. nannofossil } \\
\text { chalk }\end{array}$ & 71 & 3 & & 15 & $\begin{array}{l}7 \\
(2)\end{array}$ & \\
\hline $6-2,76$ & upper Cenomanian & 144.3 & $\begin{array}{l}\text { Foram. nannofossil } \\
\text { chalk }\end{array}$ & 93 & 3 & & 4 & & \\
\hline $6-2,84-86$ & upper Cenomanian & 144.4 & $\begin{array}{l}\text { Foram. nannofossil } \\
\text { chalk }\end{array}$ & 74 & 5 & & 10 & $\begin{array}{l}11 \\
(3)\end{array}$ & \\
\hline
\end{tabular}

Note: Dashes and blanks both mean not detected by method used.

product of volcanic glass and other pyroclastics. Thus, at least part of the black zeolitic shales of Site 551 may have originated as volcanogenic materials.

\section{DISCUSSION AND SUMMARY}

Von Rad et al. (1978) stated that the ratio of opal-CT content to diagenetic-quartz content serves as a rough measure of the mineralogical maturity of silicified sediments. The plot of this ratio versus age of Leg 80 samples shows some fluctuations from the plot of von Rad et al. (1978), particularly for the samples older than Lower Cretaceous (Fig. 3). Weakly silicified clayey sediments in which opal CT is the only diagenetic silica mineral occur in Lower Cretaceous sediments of Site 549. 


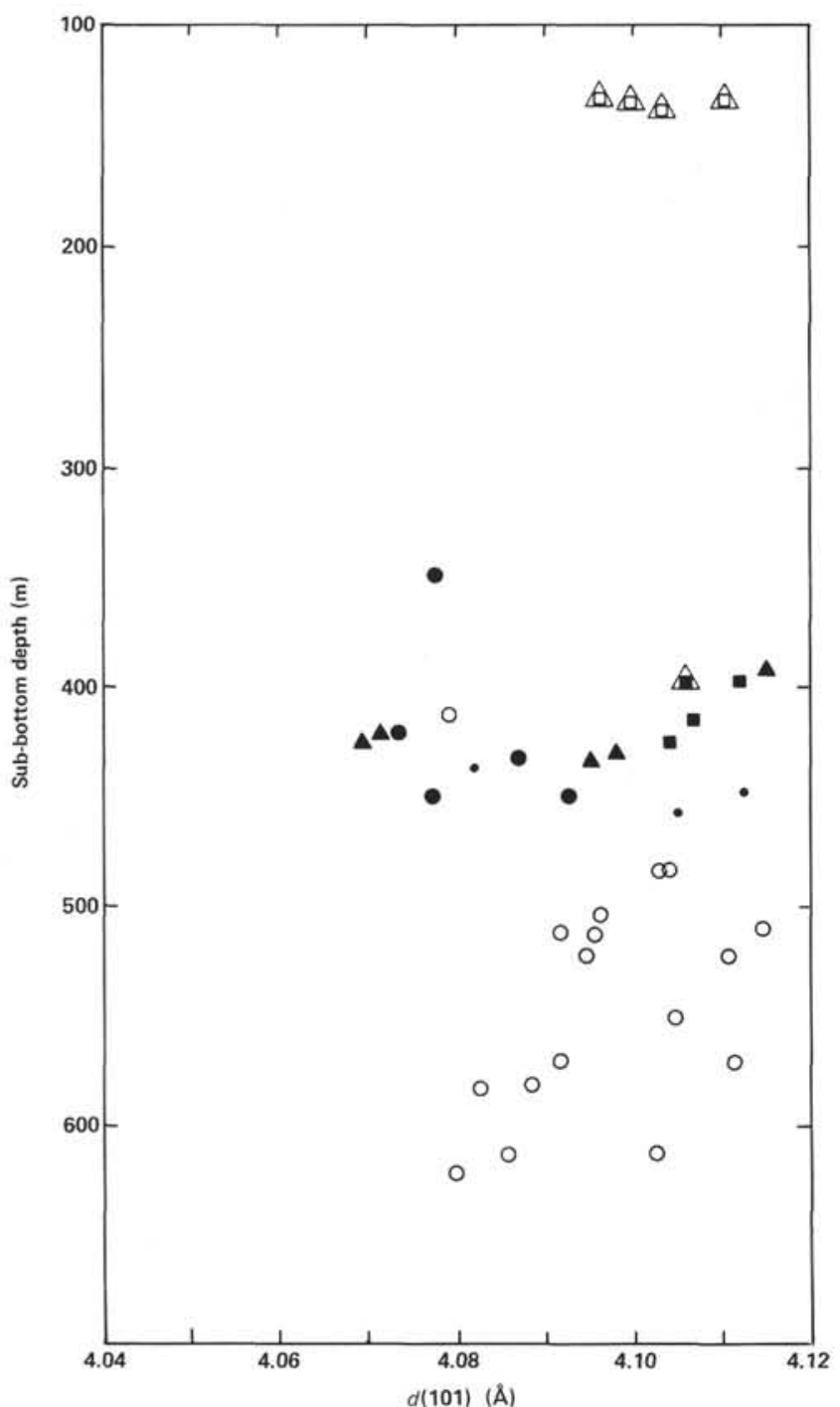

Figure 2. Relation of $d(101)$ spacing of opal CT and burial depth of silicified sediments at Leg 80 sites (symbols for silicified sediment types as in Fig. 1 legend).

Further, the ratio of opal-CT content to diagenetic-quartz content does not seem to decrease in older sediments. Rather, quartz-cherts occur in younger host sediments.

Figure 4 shows diagenetic silica facies as a function of host sediment age and sub-bottom depth. The lines dividing the mineral fields are taken from Riech and von $\operatorname{Rad}$ (1979a). The distribution pattern of Leg 80 samples is not very different from their diagenetic-mineral fields. The distribution of chert nodules from Site 548 in the field of opal A and opal CT is one anomaly; and the bedded, weakly silicified sediments from Site 549 (Lower Cretaceous), with opal CT as almost the only diagenetic silica mineral, plot in the field of opal CT and quartz and constitute another anomaly in Figure 4. This diagenetic facies indicates a little more immature stage of diagenesis than that suggested by Riech and von Rad (1979a). The anomalies may be attributed to the irregular and overlapping stages of silica diagenesis, varying between the nodular types in carbonate sediments and bedded types in clay matrices.

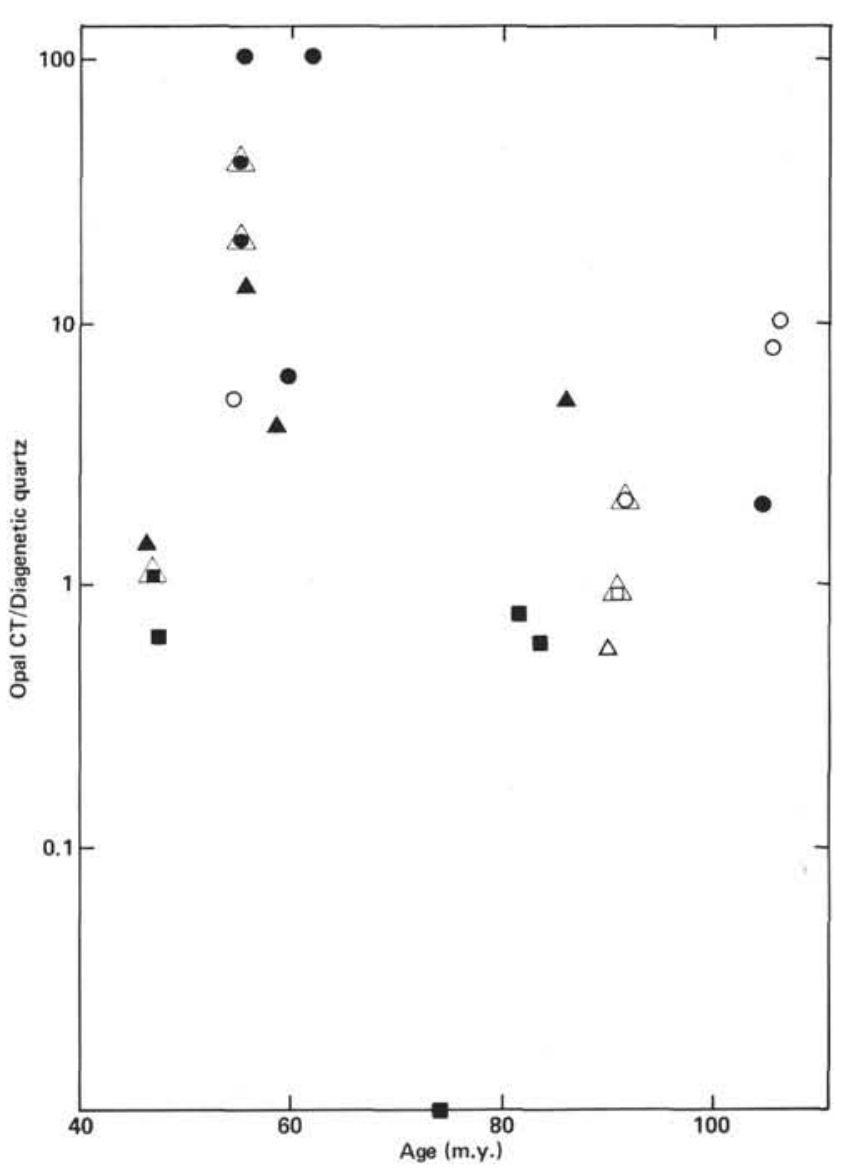

Figure 3. The ratio of opal-CT content to diagenetic-quartz content as a function of sample age for the various types of silicified sediment (symbols for silicified sediment types as in Fig. 1 legend).

As already shown by Figures 2 and 3, the nodular type of silicified sediment in carbonate-dominant host sediments tends to show rapid silica diagenesis, whereas the diagenetic change in the bedded type of silicified sediments in clayey host strata occurs more slowly. Thus, even Lower Cretaceous sediments have immature facies with high ratios of opal-CT content to diagenetic-quartz content in the case of bedded silicified sediments in clayey matrices.

Lancelot (1973) noted that foreign cations in silica solutions should be relatively abundant in clayey sediments and thereby favor the formation of diagenetic opal CT rather than quartz. With regard to opal-CT cement formation, however, this theory is not so simply applied to the bedded clayey silicified sediments of Site 549, in which silica cementation is poor.

The ordering or maturation of the opal-CT $d(101)$ spacing takes place rapidly in carbonate sediments, but is slow in clayey deposits (Fig. 2). It should be noted, however, that irregularities in the ordering or opal-CT $d$ spacings are particularly apparent in nodular-type silicified sediments in carbonate host sediments. The relation between changes in the opal-CT $d$ spacing and the silicified sediment type also suggests that quartz-chert formation is not directly related to the ordering trend of opal-CT $d$ spacings, especially for nodular-type cherts. Nodules at shallower depths have wide, disordered opal- 
Table 5. Lithofacies, chronostratigraphy, and diagenetic silica facies determined by optical and scanning-electron microscopes, selected Leg 80 silicified sediments.

\begin{tabular}{|c|c|c|c|c|c|c|c|c|c|c|}
\hline \multirow[b]{2}{*}{$\begin{array}{c}\text { Sample } \\
\text { (interval in } \mathrm{cm} \text { ) }\end{array}$} & \multirow[b]{2}{*}{$\begin{array}{l}\text { Chrono- } \\
\text { stratigraphy }\end{array}$} & \multirow{2}{*}{$\begin{array}{l}\text { Sub- } \\
\text { bottom } \\
\text { depth } \\
\text { (m) }\end{array}$} & \multirow[b]{2}{*}{ Host rock } & \multirow{2}{*}{$\begin{array}{l}\text { Lithology of } \\
\text { investigated } \\
\text { sample }\end{array}$} & \multicolumn{3}{|c|}{ Mode of occurrence of diagenetic minerals } & \multirow{2}{*}{$\begin{array}{l}\text { Diagenetic } \\
\text { silica } \\
(\%)\end{array}$} & \multirow[b]{2}{*}{$\frac{\text { Opal CT }}{\text { Diag. Qtz }}$} & \multirow[b]{2}{*}{ Remarks } \\
\hline & & & & & Fossil skeleton & $\begin{array}{l}\text { Pore filling of } \\
\text { fossil }\end{array}$ & Matrix & & & \\
\hline $548 \mathrm{~A}-18-1,70-72$ & upper Eocene & 367.7 & $\begin{array}{l}\text { Foram. nannofossil } \\
\text { chalk }\end{array}$ & $\begin{array}{l}\text { Foram. nannofossil } \\
\text { chalk }\end{array}$ & Forams. $\rightarrow$ only calcite & Forams. $\rightarrow\left\{\begin{array}{l}\text { clinoptilolite } \\
\text { clay }\end{array}\right.$ & & - & - & $\begin{array}{r}\text { Glauconite- } \\
\text { bearing }\end{array}$ \\
\hline $548 \mathrm{~A}-19-1,33-35$ & mid. Eocene & 376.9 & $\begin{array}{l}\text { Foram. nannofossil } \\
\text { chalk }\end{array}$ & $\begin{array}{l}\text { Foram. nannofossil } \\
\text { chalk }\end{array}$ & Forams. $\rightarrow$ only calcite & Forams. $\rightarrow\left\{\begin{array}{l}\text { clinoptilolite } \\
\text { clay }\end{array}\right.$ & $\begin{array}{l}\text { Pore space } \overrightarrow{ } \\
\quad \text { clinoptilolite } \\
\text { Microlens of quartz-chert }\end{array}$ & $\ll 1$ & - & \\
\hline $548 \mathrm{~A}-21-1,56-59$ & mid. Eocene & 396.1 & $\begin{array}{l}\text { Foram. nannofossil } \\
\text { chalk }\end{array}$ & $\begin{array}{l}\text { Calcareous porcel- } \\
\text { lanite nodule }\end{array}$ & $\begin{array}{l}\text { Forams. } \rightarrow \text { quartz } \\
\text { Sponge spicules } \rightarrow \text { opal CT }\end{array}$ & Forams $\rightarrow\left\{\begin{array}{l}\text { opal CT lep. } \\
\text { quartz } \\
\text { pyrite }\end{array}\right.$ & Opal CT & 64 & 1.4 & $\begin{array}{l}\text { Pyrite } \\
\text { spherules, } \\
\text { glauconite }\end{array}$ \\
\hline 548A-21-1, 93-95 & mid. Eocene & 396.5 & $\begin{array}{l}\text { Foram. nannofossil } \\
\text { chalk }\end{array}$ & $\begin{array}{l}\text { Very weakly } \\
\text { silicified } \\
\text { foram. nanno- } \\
\text { fossil chalk }\end{array}$ & Forams. $\rightarrow$ only calcite & Forams. $\rightarrow\left\{\begin{array}{l}\text { (clinoptilolite) } \\
\text { (opal CT) } \\
\text { clay }\end{array}\right.$ & $\begin{array}{l}\text { Microlens of opal CT } \\
\text { and clinoptilolite }\end{array}$ & $<1$ & $\infty$ & Glauconite \\
\hline $548 \mathrm{~A}-21-2,133-136$ & mid. Eocene & 398.4 & $\begin{array}{l}\text { Foram. nannofossil } \\
\text { chalk }\end{array}$ & $\begin{array}{l}\text { Quartzose porcel- } \\
\text { lanite nodule }\end{array}$ & $\left.\begin{array}{l}\text { Forams. } \rightarrow \text { quartz } \\
\text { Sponge } \\
\text { spicules, } \\
\text { Rads. }\end{array}\right\} \begin{array}{l}\text { opal CT } \\
\text { quartz }\end{array}$ & $\begin{array}{l}\text { Forams. } \rightarrow\left\{\begin{array}{l}\text { opal CT lep. } \\
\text { quartz } \\
\text { clinoptilolite }\end{array}\right. \\
\text { Rads. } \rightarrow\left\{\begin{array}{l}\text { opal CT } \\
\text { quartz }\end{array}\right.\end{array}$ & $\begin{array}{l}\text { Opal CT } \\
\text { Microlens of chalcedon- } \\
\quad \text { ic quartz }\end{array}$ & 56 & 1.1 & \\
\hline $548 \mathrm{~A}-22-5,62-65$ & mid. Eocene & 411.6 & $\begin{array}{l}\text { Foram. marly } \\
\text { nannofossil } \\
\text { chalk }\end{array}$ & $\begin{array}{l}\text { Foram. marly } \\
\text { nannofossil } \\
\text { chalk. }\end{array}$ & Forams. $\rightarrow$ only calcite & Forams. $\rightarrow$ clinoptilolite & $\begin{array}{l}\text { Pore space } \rightarrow \\
\text { clinoptilolite }\end{array}$ & - & - & Glauconite \\
\hline $549-18-1,22-24$ & $\begin{array}{l}\text { upper Paleo- } \\
\text { cene }\end{array}$ & 350.7 & $\begin{array}{l}\text { Siliceous nanno- } \\
\text { fossil chalk }\end{array}$ & $\begin{array}{l}\text { Weakly silicified } \\
\text { calcareous } \\
\text { porcellanite } \\
\text { nodule }\end{array}$ & $\left.\begin{array}{l}\begin{array}{l}\text { Rads. } \\
\text { Sponge } \\
\text { spicules } \\
\text { Diatoms }\end{array} \\
\text { Forams. } \rightarrow \text { only calcite }\end{array}\right\} \rightarrow$ opal CT & $\left.\begin{array}{l}\begin{array}{l}\text { Rads. } \\
\text { Sponge } \\
\text { spicules }\end{array} \\
\text { Diatoms }\end{array}\right\} \rightarrow$ opal CT & Patches of opal CT & 30 & $=100$ & \\
\hline $549-18-2,96-99$ & $\begin{array}{l}\text { upper Paleo- } \\
\text { cene }\end{array}$ & 353.0 & $\begin{array}{l}\text { Siliceous nanno- } \\
\text { fossil chalk }\end{array}$ & $\begin{array}{l}\text { Clayey concretion } \\
\text { in siliceous } \\
\text { nannofossil } \\
\text { chalk }\end{array}$ & $\left.\begin{array}{l}\text { Rads. } \\
\begin{array}{l}\text { Sponge } \\
\text { spicules } \\
\text { Diatoms }\end{array}\end{array}\right\} \rightarrow \begin{array}{l}\text { fresh, } \\
\text { partly } \\
\text { dis- } \\
\text { solved }\end{array}$ & & & - & - & \\
\hline $549-24-3,1-3$ & Campanian & 410.5 & Nannofossil chalk & $\begin{array}{l}\text { Quartz-chert } \\
\text { nodule }\end{array}$ & $\left.\begin{array}{l}\text { Forams. } \\
\text { Rads. } \\
\begin{array}{l}\text { Sponge } \\
\text { spicules } \\
\text { Diatoms }\end{array}\end{array}\right\} \begin{array}{c}\text { quartz } \\
\text { opal CT at } \\
\text { nodule } \\
\text { edge }\end{array}$ & 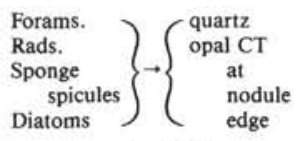 & $\begin{array}{l}\text { Quartz } \\
\text { Edge of nodule } \rightarrow \text { opal } \\
\text { CT }\end{array}$ & $=100$ & $\approx 0.01$ & $\begin{array}{l}\text { Pyrite } \\
\text { spherules }\end{array}$ \\
\hline $549-25-2,39-41$ & $\begin{array}{l}\text { Santonian- } \\
\text { Coniacian }\end{array}$ & 418.9 & Nannofossil chalk & Nannofossil chalk & $\begin{array}{l}\text { Forams. } \rightarrow \text { only calcite } \\
\text { (Rads. } \rightarrow \text { opal CT) }\end{array}$ & $\begin{array}{l}\text { Forams. } \rightarrow\left\{\begin{array}{l}\text { opal CT } \\
\text { clinoptilolite }\end{array}\right. \\
\text { (Rads. } \rightarrow \text { opal CT) }\end{array}$ & Patches of opal CT & 1 & $\infty$ & \\
\hline $549-25-2,53-55$ & $\begin{array}{l}\text { Santonian- } \\
\text { Coniacian }\end{array}$ & 419.0 & Nannofossil chalk & $\begin{array}{l}\text { Calcareous porcel- } \\
\text { laneous } \\
\text { quartz-chert }\end{array}$ & $\begin{array}{l}\text { Forams. } \rightarrow\left\{\begin{array}{l}\text { quartz } \\
\text { opal CT } \\
\text { partly } \\
\text { calcite }\end{array}\right. \\
\text { Rads. } \rightarrow\left\{\begin{array}{l}\text { quartz } \\
\text { opal CT }\end{array}\right.\end{array}$ & $\begin{array}{l}\text { Forams. } \rightarrow\left\{\begin{array}{l}\text { quartz } \\
\text { opal CT }\end{array}\right. \\
\text { Rads. } \rightarrow\left\{\begin{array}{l}\text { quartz } \\
\text { opal CT }\end{array}\right.\end{array}$ & $\begin{array}{l}\text { Opal CT and quartz as } \\
\text { matrix } \\
\text { Patches of chalcedonic } \\
\text { quartz }\end{array}$ & 88 & 0.80 & \\
\hline $549-26-1,8-9$ & Turonian & 426.6 & Nannofossil chalk & $\begin{array}{l}\text { Porcellaneous } \\
\text { quartz-chert }\end{array}$ & $\left.\begin{array}{l}\text { Rads. } \\
\text { Diatoms } \\
\begin{array}{c}\text { Sponge } \\
\text { spicules } \\
\text { Forams. }\end{array}\end{array}\right\} \rightarrow\left\{\begin{array}{l}\text { opal CT } \\
\text { quartz }\end{array}\right.$ & 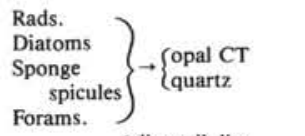 & $\begin{array}{l}\text { Opal CT and quartz as } \\
\text { matrix } \\
\text { Patches of chalcedonic } \\
\text { quartz and opal CT }\end{array}$ & 93 & 0.60 & \\
\hline $549-28-2,30-32$ & Cenomanian & 447.3 & $\begin{array}{l}\text { Foram. nannofossil } \\
\text { chalk }\end{array}$ & $\begin{array}{l}\text { Foram. nannofossil } \\
\text { chalk }\end{array}$ & $\begin{array}{l}\text { Forams. } \rightarrow \text { only calcite } \\
\text { (Rads. } \rightarrow \text { quartz) }\end{array}$ & 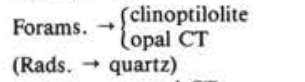 & $\begin{array}{l}\text { Patches of opal CT or } \\
\text { chalcedonic quartz }\end{array}$ & $<1$ & & Glauconite \\
\hline $549-32-1,35-37$ & mid. Albian & 483.9 & $\begin{array}{l}\text { Siliceous fossil- } \\
\text { bearing calcar- } \\
\text { eous siltstone }\end{array}$ & $\begin{array}{l}\text { Weakly silicified } \\
\text { siliceous fossil- } \\
\text { bearing calcar- } \\
\text { eous siltstone }\end{array}$ & $\begin{array}{l}\text { Forams. } \rightarrow\left\{\begin{array}{l}\text { calcite } \\
\text { partly quartz, } \\
\text { opal CT }\end{array}\right. \\
\left.\begin{array}{l}\text { Rads. } \\
\begin{array}{c}\text { Sponge } \\
\text { spicules }\end{array} \\
\text { Diatoms }\end{array}\right\} \rightarrow \text { opal CT }\end{array}$ & $\left.\begin{array}{l}\text { Forams. } \rightarrow\left\{\begin{array}{l}\text { opal CT } \\
\text { clinoptilolite } \\
\text { micritic } \\
\text { calcite }\end{array}\right. \\
\text { Rads. } \\
\left.\begin{array}{l}\text { Sponge } \\
\text { spicules }\end{array}\right\} \rightarrow \text { opal CT } \\
\text { Diatoms }\end{array}\right\}$ & $\begin{array}{l}\text { Patches of opal CT and } \\
\text { clinoptilolite } \\
\text { Opal CT matrix }\end{array}$ & 20 & $\infty$ & \\
\hline
\end{tabular}




\begin{tabular}{|c|c|c|c|c|c|c|c|c|c|c|}
\hline 549-34-1, 55-56 & mid. Albian & 503.1 & $\begin{array}{l}\text { Siliceous fossil- } \\
\text { bearing calcar- } \\
\text { eous siltstone }\end{array}$ & $\begin{array}{l}\text { Weakly silicified } \\
\text { siliceous fossil- } \\
\text { bearing calcar- } \\
\text { eous siltstone }\end{array}$ & $\left.\begin{array}{l}\text { Forams. } \rightarrow \text { partly opal CT } \\
\text { Rads. } \\
\text { Sponge } \\
\text { spicules }\end{array}\right\} \rightarrow$ opal CT & $\left.\begin{array}{l}\text { Forams. } \rightarrow\left\{\begin{array}{l}\text { opal CT } \\
\text { calcite }\end{array}\right. \\
\text { Rads. } \\
\begin{array}{c}\text { Sponge } \\
\text { spicules }\end{array}\end{array}\right\} \rightarrow$ opal CT & $\begin{array}{l}\text { Patches of opal CT and } \\
\text { clinoptilolite in pore } \\
\text { space }\end{array}$ & 7 & $\infty$ & Glauconite \\
\hline \multirow[t]{2}{*}{$549-35-1,37-39$} & \multirow[t]{2}{*}{ mid. Albian } & \multirow[t]{2}{*}{512.4} & \multirow{2}{*}{$\begin{array}{l}\text { Siliceous fossil- } \\
\text { bearing calcar- } \\
\text { eous siltstone }\end{array}$} & \multirow{2}{*}{$\begin{array}{l}\text { Porcellaneous } \\
\text { silicified } \\
\text { siliceous fossil- } \\
\text { bearing calcar- } \\
\text { eous siltstone }\end{array}$} & Forams. $\rightarrow\left\{\begin{array}{c}\text { calcite, partly } \\
\text { dissolved }\end{array}\right.$ & Forams. $\rightarrow\left\{\begin{array}{l}\text { opal CT } \\
\text { clinoptilolite }\end{array}\right.$ & \multirow{2}{*}{$\begin{array}{l}\text { Patches of opal CT and } \\
\text { clinoptilolite in pore } \\
\text { space }\end{array}$} & \multirow[t]{2}{*}{30} & \multirow[t]{2}{*}{$\infty$} & \\
\hline & & & & & $\left.\begin{array}{l}\begin{array}{l}\text { Sponge } \\
\text { spicules } \\
\text { Rads. }\end{array}\end{array}\right\} \rightarrow \begin{array}{l}\text { opal CT or } \\
\text { partly } \\
\text { dissolved }\end{array}$ & $\left.\begin{array}{l}\text { Rads. } \\
\begin{array}{c}\text { Sponge } \\
\text { spicules }\end{array}\end{array}\right\} \rightarrow \begin{array}{l}\text { opal CT } \\
\text { clinoptilo- } \\
\text { lite }\end{array}$ & & & & \\
\hline $549-36-1,15-17$ & $\begin{array}{l}\text { mid.-lower } \\
\text { Albian }\end{array}$ & 521.7 & $\begin{array}{l}\text { Laminated calcare- } \\
\text { ous siltstone }\end{array}$ & $\begin{array}{l}\text { Laminated calcare- } \\
\text { ous sandy } \\
\text { siltstone }\end{array}$ & $\begin{array}{l}\text { Forams. } \rightarrow \text { only calcite } \\
\text { Rads. } \\
\left.\begin{array}{c}\text { Sponge } \\
\text { spicules }\end{array}\right\} \rightarrow \text { opal CT }\end{array}$ & 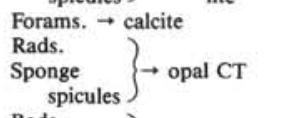 & $\begin{array}{l}\text { Clinoptilolite in pore } \\
\text { spaces }\end{array}$ & $<1$ & $\infty$ & \\
\hline $549-42-2,38-40$ & $\begin{array}{l}\text { mid.-lower } \\
\text { Albian }\end{array}$ & 570.9 & $\begin{array}{l}\text { Laminated calcare- } \\
\text { ous siltstone }\end{array}$ & $\begin{array}{l}\text { Laminated weakly } \\
\text { silicified } \\
\text { siliceous fossil- } \\
\text { bearing calcar- } \\
\text { eous siltstone }\end{array}$ & $\left.\begin{array}{l}\text { Rads. } \\
\text { Sponge } \\
\text { spicules } \\
\text { Forams. } \rightarrow \text { only calcite }\end{array}\right\} \rightarrow$ opal CT & $\begin{array}{l}\left.\begin{array}{l}\text { Rads. } \\
\text { Sponge } \\
\text { spicules }\end{array}\right\} \rightarrow \text { opal CT } \\
\text { Forams. } \rightarrow\left\{\begin{array}{l}\text { calcite } \\
\text { opal CT } \\
\text { clinntilolite }\end{array}\right.\end{array}$ & $\begin{array}{l}\text { Patches of opal CT and } \\
\text { clinoptilolite and } \\
\text { microlens of quartz }\end{array}$ & 9 & 8 & $\begin{array}{l}\text { Pyrite } \\
\text { spherules }\end{array}$ \\
\hline $549-43-3,127-130$ & $\begin{array}{l}\text { mid.-lower } \\
\text { Albian }\end{array}$ & 582.8 & $\begin{array}{l}\text { Laminated calcare- } \\
\text { ous siltstone }\end{array}$ & $\begin{array}{l}\text { Laminated weakly } \\
\text { silicified } \\
\text { siliceous fossil- } \\
\text { bearing calcar- } \\
\text { eous siltstone }\end{array}$ & $\begin{array}{l}\text { Forams. } \rightarrow\left\{\begin{array}{c}\text { calcite } \\
\text { partly } \\
\text { quartz and } \\
\text { opal CT }\end{array}\right. \\
\left.\begin{array}{l}\text { Rads. } \\
\begin{array}{c}\text { Sponge } \\
\text { spicules }\end{array}\end{array}\right\} \rightarrow \text { opal CT }\end{array}$ & $\begin{array}{l}\text { Forams. } \rightarrow\left\{\begin{array}{l}\text { clinoptilolite } \\
\text { opal CT } \\
\text { clinoptilolite } \\
\text { calcite }\end{array}\right. \\
\left.\begin{array}{l}\text { Rads. } \\
\text { Sponge } \\
\text { spicules }\end{array}\right\} \rightarrow\left\{\begin{array}{c}\text { opal CT } \\
\text { clinoptilo- } \\
\text { lite }\end{array}\right.\end{array}$ & $\begin{array}{l}\text { Patches of opal CT and } \\
\text { clinoptilolite in pore } \\
\text { spaces }\end{array}$ & 9 & 8 & \\
\hline $549-44-3,141-143$ & $\begin{array}{l}\text { mid.-lower } \\
\text { Albian }\end{array}$ & 592.4 & $\begin{array}{l}\text { Laminated calcare- } \\
\text { ous siltstone }\end{array}$ & $\begin{array}{l}\text { Laminated sili- } \\
\text { ceous fossil- } \\
\text { bearing calcar- } \\
\text { eous siltstone }\end{array}$ & $\left.\begin{array}{l}\text { Forams. } \rightarrow \text { calcite } \\
\text { Rads. } \\
\begin{array}{c}\text { Sponge } \\
\text { spicules }\end{array}\end{array}\right\} \rightarrow$ opal CT & Forams. $\rightarrow\left\{\begin{array}{l}\text { opal CT } \\
\text { clinoptilolite } \\
\text { calcite } \\
\text { pyrite }\end{array}\right.$ & $\begin{array}{l}\text { Patches of opal CT and } \\
\text { clinoptilolite in pore } \\
\text { spaces }\end{array}$ & $<1$ & $\infty$ & \\
\hline & & & & & & $\left.\begin{array}{l}\text { Rads. } \\
\text { Sponge } \\
\text { spicules }\end{array}\right\} \rightarrow\left\{\begin{array}{c}\text { opal CT } \\
\text { clinoptilo- } \\
\text { lite }\end{array}\right.$ & & & & \\
\hline $549-47-4,14-17$ & $\begin{array}{l}\text { mid.-lower } \\
\text { Albian }\end{array}$ & 621.2 & $\begin{array}{l}\text { Laminated calcare- } \\
\text { ous sandy } \\
\text { siltstone }\end{array}$ & $\begin{array}{l}\text { Laminated very } \\
\text { weakly silici- } \\
\text { fied calcareous } \\
\text { sandy siltstone }\end{array}$ & $\left.\begin{array}{l}\text { Forams. } \rightarrow \text { only calcite } \\
\text { Sponge } \\
\text { spicules } \\
\text { Rads. }\end{array}\right\} \rightarrow$ opal CT & 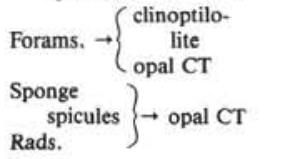 & $\begin{array}{l}\text { Patches of chalcedonic } \\
\text { quartz }\end{array}$ & 4 & $\approx 10$ & Glauconite \\
\hline $549-47-4,24-26$ & $\begin{array}{l}\text { mid.-lower } \\
\text { Albian }\end{array}$ & 621.3 & $\begin{array}{l}\text { Calcareous silt- } \\
\text { stone }\end{array}$ & $\begin{array}{l}\text { Sparitic calcareous } \\
\text { siltstone }\end{array}$ & Rads. $\rightarrow$ dissolved & & $\begin{array}{l}\text { Sparitic fine calcite } \\
\text { cement }\end{array}$ & - & - & \\
\hline $550-35-5,69-71$ & $\begin{array}{l}\text { upper Paleo- } \\
\text { cene }\end{array}$ & 419.7 & $\begin{array}{l}\text { Siliceous marly } \\
\text { nannofossil } \\
\text { chalk }\end{array}$ & $\begin{array}{l}\text { Nannofossil- } \\
\text { bearing sili- } \\
\text { ceous claystone }\end{array}$ & $\left.\begin{array}{l}\begin{array}{l}\text { Sponge } \\
\text { spicules } \\
\text { Rads. } \\
\text { Diatoms }\end{array}\end{array}\right\} \rightarrow \begin{array}{c}\text { fresh or } \\
\text { dissolved }\end{array}$ & & & - & - & \\
\hline $550-35-5,87-89$ & $\begin{array}{l}\text { upper Paleo- } \\
\text { cene }\end{array}$ & 419.9 & $\begin{array}{l}\text { Siliceous marly } \\
\text { nannofossil } \\
\text { chalk }\end{array}$ & $\begin{array}{l}\text { Foram. marly } \\
\text { nanno. chalk } \\
\text { and siliceous } \\
\text { claystone }\end{array}$ & $\left.\begin{array}{l}\begin{array}{l}\text { Sponge } \\
\text { spicules } \\
\text { Rads. } \\
\text { Diatoms }\end{array}\end{array}\right\} \rightarrow \begin{array}{r}\text { partly dis- } \\
\text { solved or } \\
\text { opal CT }\end{array}$ & Rads. $\rightarrow\left\{\begin{array}{l}\text { clinoptilolite } \\
\text { opal CT }\end{array}\right.$ & $\begin{array}{l}\text { Pore space } \rightarrow \\
\text { clinoptilolite }\end{array}$ & $<1$ & $\infty$ & \\
\hline $550-36-1,12-15$ & $\begin{array}{l}\text { upper Paleo- } \\
\text { cene }\end{array}$ & 422.6 & $\begin{array}{l}\text { Calcareous clay- } \\
\text { stone }\end{array}$ & $\begin{array}{l}\text { Laminated calcare- } \\
\text { ous mudstone }\end{array}$ & 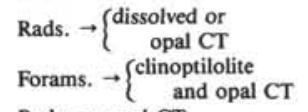 & $\begin{array}{l}\text { Rads. } \rightarrow \text { opal CT } \\
\text { Forams. } \rightarrow \text { opal CT }\end{array}$ & $\begin{array}{l}\text { Dolomite rhombic } \\
\text { crystals }\end{array}$ & $<1$ & $\infty$ & \\
\hline $550-36-1,132-135$ & $\begin{array}{l}\text { upper Paleo- } \\
\text { cene }\end{array}$ & 423.8 & $\begin{array}{l}\text { Calcareous clay- } \\
\text { stone }\end{array}$ & $\begin{array}{l}\text { Porcellaneous } \\
\text { dolomite } \\
\text { nodule }\end{array}$ & $\begin{array}{l}\text { Rads. } \rightarrow \text { opal CT } \\
\text { Sponge } \\
\text { spicules }\} \rightarrow \text { opal CT } \\
\text { (Forams. } \rightarrow \text { only calcite) }\end{array}$ & $\begin{array}{l}\text { Rads. } \rightarrow\left\{\begin{array}{l}\text { quartz } \\
\text { opal CT }\end{array}\right. \\
\text { (Forams. } \rightarrow\left\{\begin{array}{l}\text { quartz } \\
\text { opal CT) }\end{array}\right.\end{array}$ & $\begin{array}{l}\text { Distinct rhombic } \\
\text { dolomite crystals } \\
\text { Opal CT matrix } \\
\text { Veins of chalcedonic } \\
\text { quartz and opal CT } \\
\text { lep. }\end{array}$ & 21 & 20 & \\
\hline $550-36-2,41-45$ & $\begin{array}{l}\text { upper Paleo- } \\
\text { cene }\end{array}$ & 424.4 & $\begin{array}{l}\text { Calcareous mud- } \\
\text { stone }\end{array}$ & $\begin{array}{l}\text { Dolomitic porcel- } \\
\text { lanite nodule }\end{array}$ & $\begin{array}{l}\text { Rad. } \rightarrow \text { opal CT } \\
\left.\begin{array}{c}\text { Sponges } \\
\text { spicules }\end{array}\right\} \rightarrow \begin{array}{c}\text { quartz } \\
\text { opal CT }\end{array} \\
\text { (Forams. } \rightarrow\left\{\begin{array}{c}\text { partly } \\
\text { quartz) }\end{array}\right.\end{array}$ & $\begin{array}{l}\text { Rads. } \rightarrow\left\{\begin{array}{l}\text { opal CT } \\
\text { quartz }\end{array}\right. \\
\left.\begin{array}{l}\text { Sponge } \\
\text { spicules }\end{array}\right\} \rightarrow \text { opal CT } \\
\text { (Forams. } \rightarrow\left\{\begin{array}{l}\text { opal CT } \\
\text { dolomite) }\end{array}\right.\end{array}$ & $\begin{array}{l}\text { Opal CT matrix } \\
\text { Rhombic dolomite } \\
\text { crystals } \\
\text { Veins of chalcedonic } \\
\text { quartz and opal CT }\end{array}$ & 72 & 13.4 & \\
\hline
\end{tabular}


Table 5. (Continued).

\begin{tabular}{|c|c|c|c|c|c|c|c|c|c|c|}
\hline \multirow[b]{2}{*}{$\begin{array}{c}\text { Sample } \\
\text { (interval in } \mathrm{cm} \text { ) }\end{array}$} & \multirow[b]{2}{*}{$\begin{array}{l}\text { Chrono- } \\
\text { stratigraphy }\end{array}$} & \multirow{2}{*}{$\begin{array}{l}\text { Sub- } \\
\text { bottom } \\
\text { depth } \\
\text { (m) }\end{array}$} & \multirow[b]{2}{*}{ Host rock } & \multirow{2}{*}{$\begin{array}{l}\text { Lithology of } \\
\text { investigated } \\
\text { sample }\end{array}$} & \multicolumn{3}{|c|}{ Mode of occurrence of diagenetic minerals } & \multirow{2}{*}{$\begin{array}{l}\text { Diagenetic } \\
\text { silica } \\
(\%)\end{array}$} & \multirow[b]{2}{*}{$\frac{\text { Opal CT }}{\text { Diag. Qtz }}$} & \multirow[b]{2}{*}{ Remarks } \\
\hline & & & & & Fossil skeleton & $\begin{array}{l}\text { Pore filling of } \\
\text { fossil }\end{array}$ & Matrix & & & \\
\hline $550-37-1,0-3$ & $\begin{array}{l}\text { upper Paleo- } \\
\text { cene }\end{array}$ & 432.0 & Nannofossil chalk & $\begin{array}{l}\text { Calcareous porcel- } \\
\text { lanite nodule }\end{array}$ & $\begin{array}{l}\text { Rads. } \rightarrow\left\{\begin{array}{l}\text { opal CT } \\
\text { quartz }\end{array}\right. \\
\left.\begin{array}{c}\text { Sponge } \\
\text { spicules }\end{array}\right\} \rightarrow\left\{\begin{array}{l}\text { opal CT } \\
\text { quartz }\end{array}\right. \\
\text { Forams. } \rightarrow \text { quartz }\end{array}$ & $\begin{array}{l}\text { Rads. } \rightarrow\left\{\begin{array}{l}\text { quartz } \\
\text { opal CT }\end{array}\right. \\
\left.\begin{array}{c}\text { Sponge } \\
\text { spicules }\end{array}\right\} \rightarrow\left\{\begin{array}{l}\text { quartz } \\
\text { opal CT }\end{array}\right. \\
\text { Forams. } \rightarrow\left\{\begin{array}{l}\text { quartz } \\
\text { opal CT }\end{array}\right.\end{array}$ & Opal CT matrix & 75 & 4 & \\
\hline $550-38-6,104-106$ & $\begin{array}{l}\text { lower Paleo- } \\
\text { cene }\end{array}$ & 450.1 & Nannofossil chalk & $\begin{array}{l}\text { Weakly silicified } \\
\text { siliceous fossil- } \\
\text { bearing nanno- } \\
\text { fossil chalk }\end{array}$ & $\left.\begin{array}{l}\text { Rads. } \\
\text { Diatoms } \\
\begin{array}{c}\text { Sponge } \\
\text { spicules }\end{array}\end{array}\right\} \rightarrow\left\{\begin{array}{l}\text { opal CT } \\
\text { clinoptilo- } \\
\text { lite }\end{array}\right.$ & $\left.\begin{array}{l}\text { Rads. } \\
\text { Diatoms } \\
\begin{array}{c}\text { Sponge } \\
\text { spicules }\end{array}\end{array}\right\} \rightarrow\left\{\begin{array}{c}\text { opal CT } \\
\text { clinotilo- } \\
\text { lite }\end{array}\right.$ & 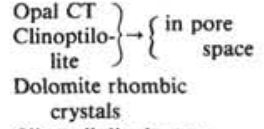 & 23 & $\infty$ & \\
\hline $550-39-2,35-37$ & $\begin{array}{l}\text { lower Paleo- } \\
\text { cene }\end{array}$ & 452.9 & $\begin{array}{l}\text { Marly nannofossil } \\
\text { chalk }\end{array}$ & $\begin{array}{l}\text { Marly siliceous } \\
\text { nannofossil } \\
\text { chalk }\end{array}$ & $\left.\begin{array}{l}\text { Rads. } \\
\text { Diatoms } \\
\begin{array}{l}\text { Sponge } \\
\text { spicules }\end{array} \\
\text { Forams. } \rightarrow \text { only calcite }\end{array}\right\} \rightarrow$ clinoptilolite & 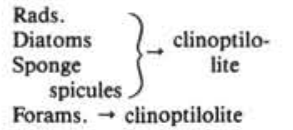 & $\begin{array}{l}\text { Clinoptilolite in pore } \\
\text { space }\end{array}$ & - & - & \\
\hline $551-5-2,3-5$ & $\begin{array}{l}\text { lower Turoni- } \\
\text { an }\end{array}$ & 134.0 & Nannofossil chalk & $\begin{array}{l}\text { Marly silicified } \\
\text { nannofossil } \\
\text { chalk with } \\
\text { porcellaneous } \\
\text { chert patches }\end{array}$ & $\begin{array}{l}\text { Forams. } \rightarrow \text { quartz } \\
\text { Rads. } \rightarrow\left\{\begin{array}{l}\text { opal CT lep. } \\
\text { quartz }\end{array}\right.\end{array}$ & $\begin{array}{l}\text { Forams. } \rightarrow\left\{\begin{array}{l}\text { opal CT } \\
\text { lep. } \\
\text { quartz }\end{array}\right. \\
\text { Rads. } \rightarrow\left\{\begin{array}{l}\text { opal CT lep. } \\
\text { quartz }\end{array}\right.\end{array}$ & $\begin{array}{l}\text { Microlenses or patches } \\
\text { of chalcedonic } \\
\text { quartz-chert with } \\
\text { opal-CT lepisphere } \\
\text { Opal CT }\end{array}$ & 56 & 0.56 & \\
\hline $551-5-2,50-53$ & $\begin{array}{l}\text { lower Turoni- } \\
\text { an }\end{array}$ & 134.5 & Nannofossil chalk & $\begin{array}{l}\text { Marly silicified } \\
\text { nannofossil } \\
\text { chalk with } \\
\text { porcellaneous } \\
\text { chert patches }\end{array}$ & Rads. $\rightarrow\left\{\begin{array}{l}\text { opal CT } \\
\text { quartz }\end{array}\right.$ & Rads. $\rightarrow\left\{\begin{array}{l}\text { quartz } \\
\text { opal CT }\end{array}\right.$ & $\begin{array}{l}\text { Opal CT } \\
\text { Microlenses or patches } \\
\text { of chalcedonic } \\
\text { quartz-chert bearing } \\
\text { opal CT lep. }\end{array}$ & 52 & 0.93 & \\
\hline $551-5-2,57-78$ & $\begin{array}{l}\text { lower Turoni- } \\
\text { an }\end{array}$ & 134.6 & $\begin{array}{l}\text { Occurred at the } \\
\text { boundary of } \\
\text { nannofossil } \\
\text { marly chalk } \\
\text { and black } \\
\text { shale }\end{array}$ & $\begin{array}{l}\text { Green and black } \\
\text { granular } \\
\text { porcellaneous } \\
\text { cherty mud }\end{array}$ & $\begin{array}{l}\text { Rads. } \rightarrow\left\{\begin{array}{l}\text { opal CT } \\
\text { quartz } \\
\text { qurale) }\end{array}\right. \\
\text { (Forams. } \rightarrow \text { calcite) }\end{array}$ & Rads. $\rightarrow\left\{\begin{array}{l}\text { quartz } \\
\text { opal CT } \\
\text { clinoptilolite }\end{array}\right.$ & $\begin{array}{l}\text { Patches of opal CT and } \\
\text { chalcedonic quartz } \\
\text { Clinoptilolite in matrix } \\
\text { and also as patches } \\
\text { Clays as matrix }\end{array}$ & 45 & 2 & $\begin{array}{l}\text { Pyrite } \\
\text { spherules }\end{array}$ \\
\hline $551-5-2,79-81$ & $\begin{array}{l}\text { lower Turoni- } \\
\text { an }\end{array}$ & 134.8 & Black shale & $\begin{array}{l}\text { Black zeolitic } \\
\text { carbonaceous } \\
\text { mudstone with } \\
\text { laminations of } \\
\text { white zeolitic } \\
\text { clay }\end{array}$ & (Rads. $\rightarrow$ opal CT or A) & (Rads. $\rightarrow$ clinoptilolite) & $\begin{array}{l}\text { Clinoptilolite as matrix } \\
\text { and also patches } \\
\text { Phillipsite euhedral } \\
\text { crystals } \\
\text { (Chalcedonic quartz } \\
\text { patches) }\end{array}$ & $\ll 1$ & - & $\begin{array}{l}\text { Pyrite } \\
\text { spherules }\end{array}$ \\
\hline $551-5-2,101-103$ & $\begin{array}{l}\text { lower Turoni- } \\
\text { an }\end{array}$ & 135.0 & Black shale & $\begin{array}{l}\text { Black zeolitic } \\
\text { carbonaceous } \\
\text { mudstone with } \\
\text { laminations of } \\
\text { white zeolitic } \\
\text { clay }\end{array}$ & $\begin{array}{l}\text { Rads. } \rightarrow\left\{\begin{array}{l}\text { opal CT } \\
\text { quartz }\end{array}\right. \\
\text { Forams. } \rightarrow \text { calcite only }\end{array}$ & Rads. $\rightarrow\left\{\begin{array}{l}\text { quartz } \\
\text { opal CT }\end{array}\right.$ & $\begin{array}{l}\text { Phillipsite euhedral } \\
\text { crystals } \\
\text { Clinoptilolite as matrix } \\
\text { and also patches } \\
\text { Clays as matrix }\end{array}$ & $\ll 1$ & - & $\begin{array}{l}\text { Glauconite } \\
\text { pyrite } \\
\text { spherules }\end{array}$ \\
\hline $551-6-1,10-12$ & $\begin{array}{l}\text { upper Ceno- } \\
\text { manian }\end{array}$ & 142.2 & $\begin{array}{l}\text { Foram. nannofossil } \\
\text { chalk }\end{array}$ & $\begin{array}{l}\text { Foram. nannofossil } \\
\text { chalk }\end{array}$ & Forams. $\rightarrow$ calcite only & Forams. $\rightarrow\left\{\begin{array}{l}\text { opal CT } \\
\text { clinoptilolite }\end{array}\right.$ & & $\ll 1$ & - & \\
\hline $551-6-2,82-84$ & $\begin{array}{l}\text { upper Ceno- } \\
\text { manian }\end{array}$ & 144.4 & $\begin{array}{l}\text { Foram. nannofossil } \\
\text { chalk }\end{array}$ & $\begin{array}{l}\text { Foram. nannofossil } \\
\text { chalk }\end{array}$ & Forams. $\rightarrow$ calcite only & Forams. $\rightarrow$ clinoptilolite & & - & - & \\
\hline
\end{tabular}

Notes: Where diagenetic minerals are referred to as "only calcite," no siliceous fossils were discovered. Quantities called infinite are approximations. Dashes and blanks both mean not detected by method used. 


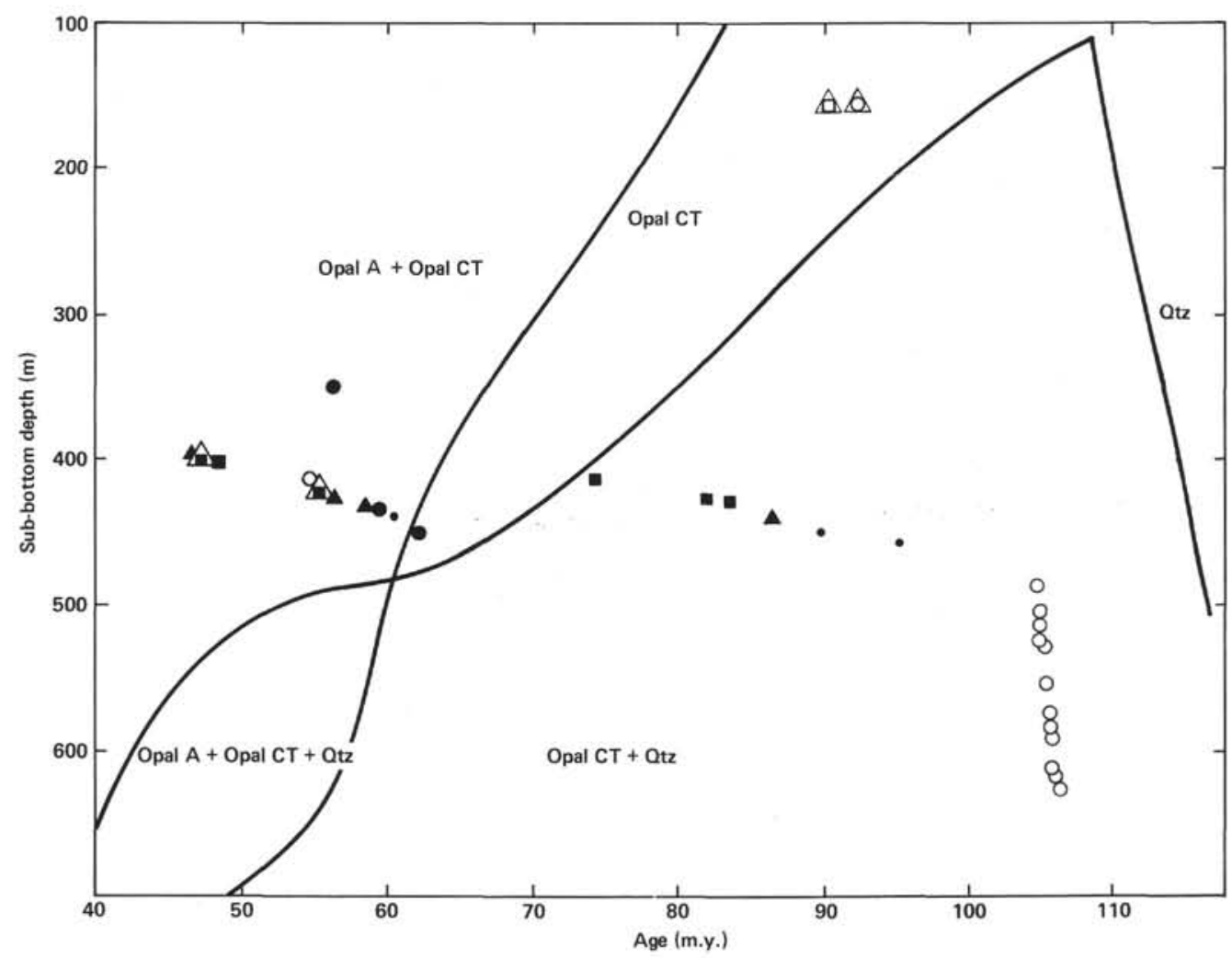

Figure 4. The relation of burial depth to sample age for the various types of silicified sediments. The fields of diagenetic silica minerals are taken from Riech and von Rad (1979a). (Symbols for silicified sediment types as in Fig. 1 legend.)

CT $d$ spacings, yet indicate mature quartz chertification and a higher diagenetic quartz content. This is inconsistent with the generally accepted concept of diagenetic quartz formation after maturation or ordering of opal CT. The diagenesis of silicified sediments seems to progress more regularly with depth in the case of clayey sediments, leading to diagenetic quartz formation at deeper levels.

The most peculiar occurrence of diagenetically silicified sediments at Leg 80 sites is the discordantly shallower one in Section 551-5-2, where higher-grade diagenetically silicified sediments were recovered. This can be explained by erosion of as much as $300 \mathrm{~m}$ of overlying sediments at Site 551. Silicified sediments that underwent diagenesis at deeper levels are now situated close to the seafloor.

Clinoptilolite occurrences span the zones of diagenetic silica mineral formation. Riech (1980) stated, however, that clinoptilolite forms later than opal CT. The discrepancy between that statement and the Leg 80 results may indicate that the formation of clinoptilolite and the diagenesis of silica are essentially independent phenomena. Further study is necessary to resolve this question.

\section{ACKNOWLEDGMENTS}

I gratefully acknowledge the kind encouragement and support of Professor Noriyuki Nasu and Dr. Hideo Kagami of the Ocean Research Institute, University of Tokyo, during this study. On board the Glomar Challenger, Dr. D. Waples of Mobile Research and Develop- ment Corporation offered me some samples for XRD analysis. $\mathrm{H}$. Ueda of Shizuoka University helped me with operation of the scanning-electron microscope. Dr. R. Matsumoto of the University of Tokyo gave critical comments on this paper. Dr. P. Loubere of Northern Illinois University kindly corrected the manuscript. Professor G. R. Heath of Oregon State University and Hakuyu Okada of Shizuoka University reviewed and carefully corrected the manuscript. I am greatly indebted to these people. This study has been supported by a grant-inaid from the Ministry of Education, Science, and Culture (Monbusho) and the Japanese IPOD Committee.

\section{REFERENCES}

Garrison, R. E., Rowland, S. M., Horan, L. J., and Moore, J. C., 1975. Petrology of siliceous rocks recovered from marginal seas of the western Pacific, Leg 31, Deep Sea Drilling Project. In Karig, D. E., Ingle, J. C., et al., Init. Repts. DSDP, 31: Washington (U.S. Govt. Printing Office), 519-529.

Grehin, V. I., Pisciotto, K. A., Mahoney, J. J., and Gordeeva, S. N., 1981. Neogene siliceous sediments and rocks off southern California and Baja California, Deep Sea Drilling Project Leg 63. In Yeats, R. S., Haq, B. U., et al., Init. Repts. DSDP, 63: Washington (U.S. Govt. Printing Office), 579-593.

Heath, G. R., 1973. Cherts from the eastern Pacific, Leg 16, Deep Sea Drilling Project. In van Andel, T. H., Heath, G. R., et al., Init. Repts. DSDP, 16: Washington (U.S. Govt. Printing Office), 609-613.

Heath, G. R., and Moberly, R. M., 1971. Cherts from the western Pacific, Leg 7, Deep Sea Drilling Project. In Winterer, E. L., Riedel, W. R., et al., Init. Repts. DSDP, 7, Pt. 2: Washington (U.S. Govt. Printing Office), 991-1007.

Hein, J. R., Vallier, T. L., and Allan, M. A., 1981. Chert petrology and geochemistry, Mid-Pacific Mountains and Hess Rise, Deep Sea Drilling Project Leg 62. In Thiede, J., Vallier, T. L., et al., Init. Repts. DSDP, 62: Washington (U.S. Govt. Printing Office), 711-748. 
Iijima, A., Matsumoto, R., and Tada, R., 1980. Zeolite and silica diagenesis and sandstone petrography at Sites $\mathbf{4 3 8}$ and 439 off Sanriku, northwest Pacific, Leg 57, Deep Sea Drilling Project. In Scientific Party, Init. Repts. DSDP, 56, 57, Pt. 2: Washington (U.S. Govt. Printing Office), 1143-1158.

Jones, J. B., and Segnit, E. R., 1971. The nature of opal, I. Nomenclature and constituent phases. J. Geol. Soc. Australia, 18:57-68.

Kagami, H., 1979. Translation of opaline silica in sediments from Bay of Biscay and Rockall Bank. In Montadert, L., Roberts, D. G., et al., Init. Repts. DSDP, 48: Washington (U.S. Govt. Printing Office), 757-764.

Keene, J. B., 1975. Cherts and porcellanites from the North Pacific, DSDP Leg 32. In Larson, R. L., Moberly, R., et al., Init. Repts. $D S D P, 32$ : Washington (U.S. Govt. Printing Office), 429-507.

Kelts, K., 1976. Summary of chert occurrences from Line Islands Sites $314,315,316$, DSDP Leg 33. In Schlanger, S. O., Jackson, E. D., et al., Init. Repts. DSDP, 33: Washington (U.S. Govt. Printing Office), 855-863.

Lancelot, Y., 1973. Chert and silica diagenesis in sediments from the central Pacific. In Winterer, E. L., Ewing, J. I., et al., Init. Repts. DSDP, 17: Washington (U.S. Govt. Printing Office), 377-405.

Mitsui, K., 1975. Diagenetic alteration of some minerals in argillaceous sediments in western Hokkaido, Japan. Sci. Rep. Res. Inst. Tohoku Univ., 13:13-65.

Murata, K. J., and Larson, R. R., 1975. Diagenesis of Miocene siliceous shales, Temblor Range, California. J. Res. U.S. Geol. Surv., 3:553-566.

Pisciotto, K. A., 1980. Chert and porcellanite from Deep Sea Drilling Project Site 436, northwest Pacific. In Scientific Party, Init. Repts. DSDP, 56, 57, Pt. 2: Washington (U.S. Govt. Printing Office), 1133-1142.
Riech, V., 1979. Diagenesis of silica, zeolites and phyllosilicates at Sites 397 and 398. In von Rad, U., Ryan, W. B. F., et al., Init. Repts. DSDP, 47, Pt. 1: Washington (U.S. Govt. Printing Office), 741-759.

1980. Diagenesis of siliceous sediments, porcellanites, and cherts of the Moroccan Basin, Deep Sea Drilling Project Sites 370 415, and 416. In Lancelot, Y., Winterer, E. L., et al., Init. Repts. $D S D P, 50$ : Washington (U.S. Govt. Printing Office), 725-731.

1981. Siliceous sediments from the Nauru Basin: diagenetic alteration of biogenic opal and authigenesis of silica and silicates. In Larson, R. L., Schlanger, S. O., et al., Init. Repts. DSDP, 61: Washington (U.S. Govt. Printing Office), 523-531.

Riech, V., and von Rad, U., 1979a. Silica diagenesis in the Atlantic Ocean: diagenetic potential and transformations. In Talwani, M., et al. (Eds.), Deep Drilling Results in the Atlantic Ocean: Continental Margins and Paleoenvironment. Am. Geophys. Union, Maurice Ewing Series 3:315-340.

1979b. Eocene porcellanites and Early Cretaceous cherts from the western North Atlantic Basin. In Tucholke, B. E., Vogt, P. R., et al., Init. Repts. DSDP, 43: Washington (U.S. Govt. Printing Office), 437-455.

von Rad, U., Riech, V., and Rosch, H., 1978. Silica diagenesis in continental margin sediments off northwest Africa. In Lancelot, Y., Seibold, E., et al., Init. Repts. DSDP, 41: Washington (U.S. Govt. Printing Office), 879-905.

Date of Initial Receipt: January 28, 1983

Date of Acceptance: November 23, 1983 

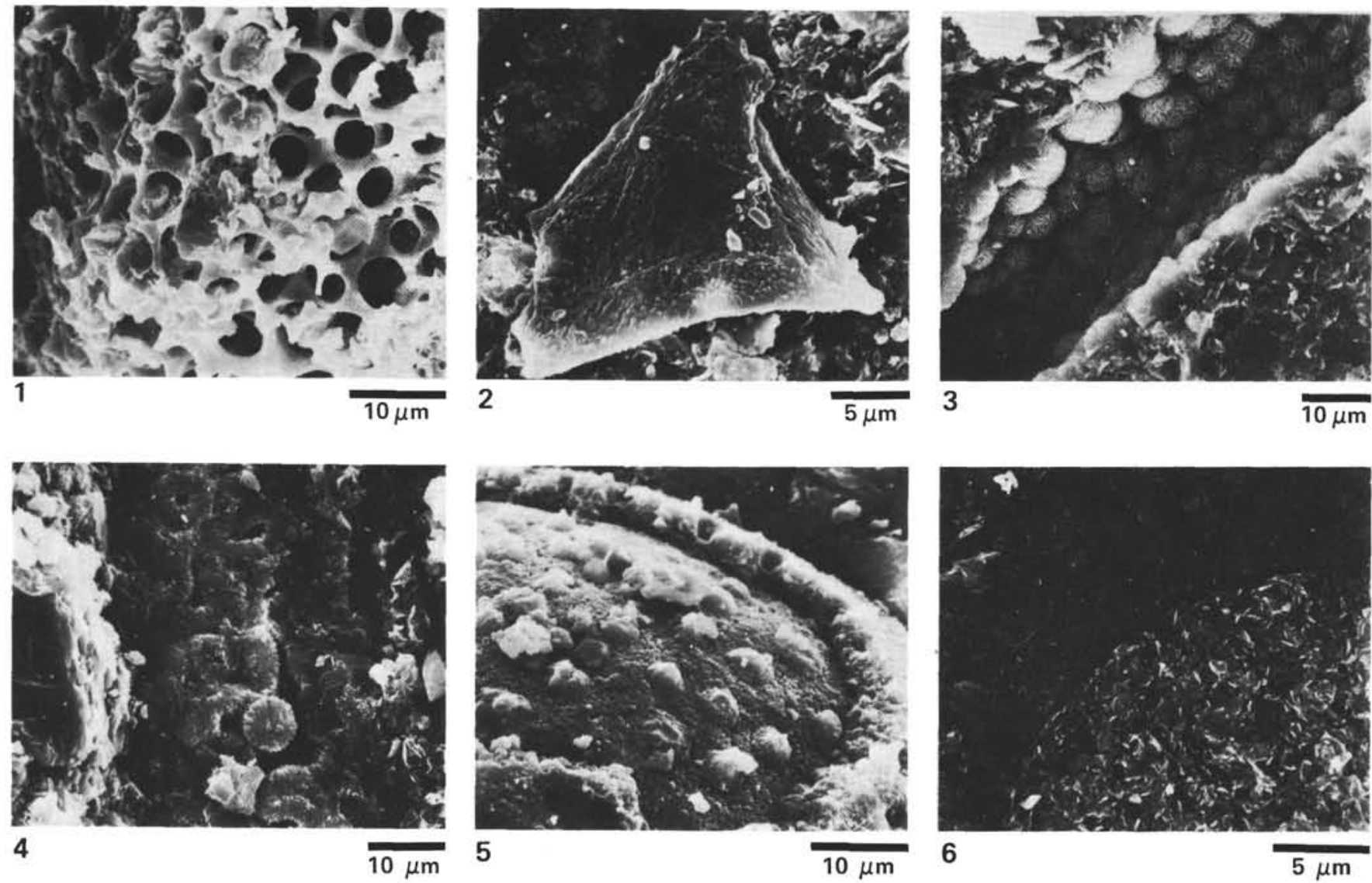

Plate 1. Scanning-electron micrograph of silica diagenetic change of biogenic skeletons and precipitation of silica minerals in pore spaces. 1 . Sample 549-18-2, 96-99 cm (upper Paleocene, sub-bottom depth $353 \mathrm{~m}, \times 2200$ ), well-preserved radiolarian skeleton, almost fresh but weakly dissolved. 2, 6. Sample 548A-21-2, 133-136 cm (middle Eocene, sub-bottom depth $398 \mathrm{~m}$ ), (2) $\times 3600$, a fragment of sponge spicule with distinct dissolution pits on the surface, (6) $\times 4400$, foraminiferal test replaced by quartz, with sediment filling the inner pore space. 3. Sample 549-35-1, 37-39 cm (middle Albian, sub-bottom depth $512 \mathrm{~m}, \times 1500$ ), opal-CT lepispheres precipitated as thin wall, probably on the trace of sponge spicule. 4. Sample 549-42-2, 38-40 cm (lower Albian, sub-bottom depth $571 \mathrm{~m}, \times 1800$ ), opal-CT lepispheres precipitated in pore space in clayey sediment matrix. 5. Sample 549-32 (top), top-situated nodule (middle Albian?, sub-bottom depth $484 \mathrm{~m}, \times 2200$ ), diagenetic quartz precipitated in the pore space of a foraminifer, the mold of the inner texture clearly evident. 


\section{K. OTSUKA}

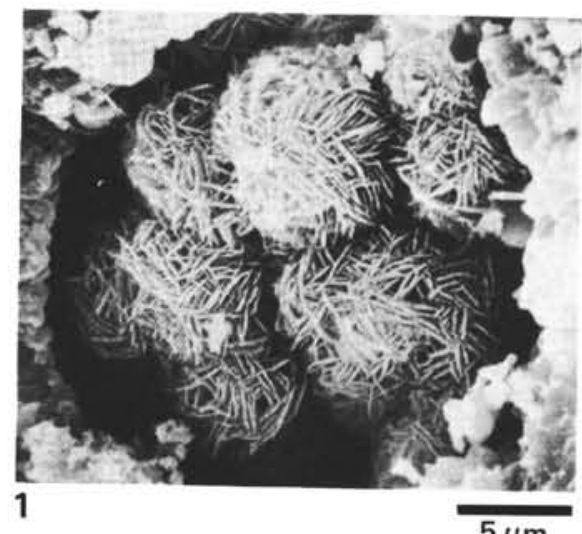

$5 \mu \mathrm{m}$

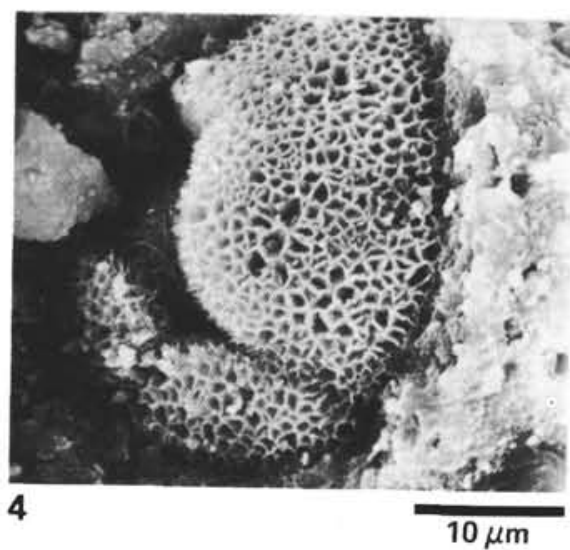

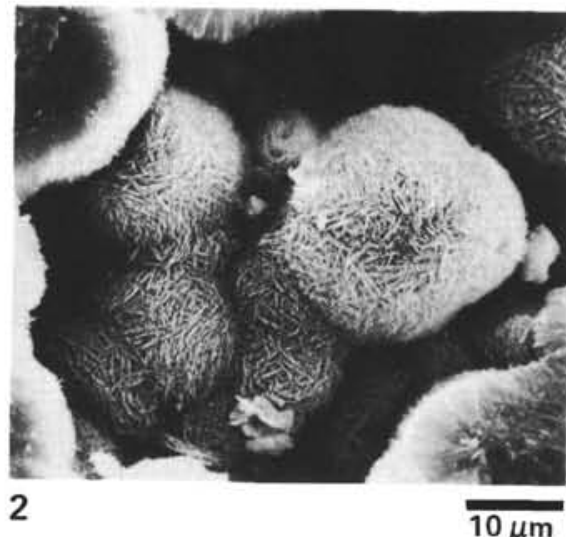

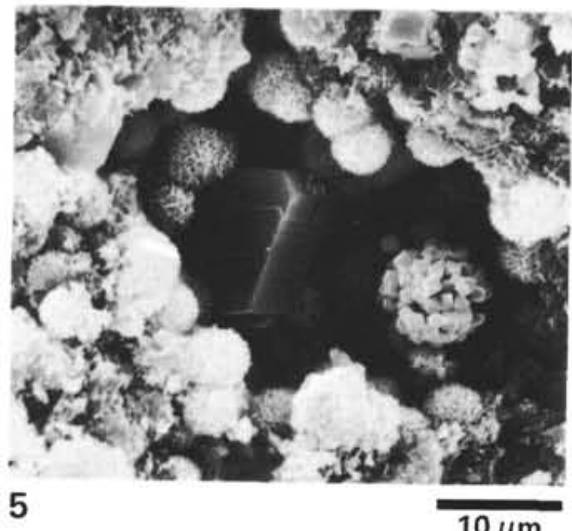

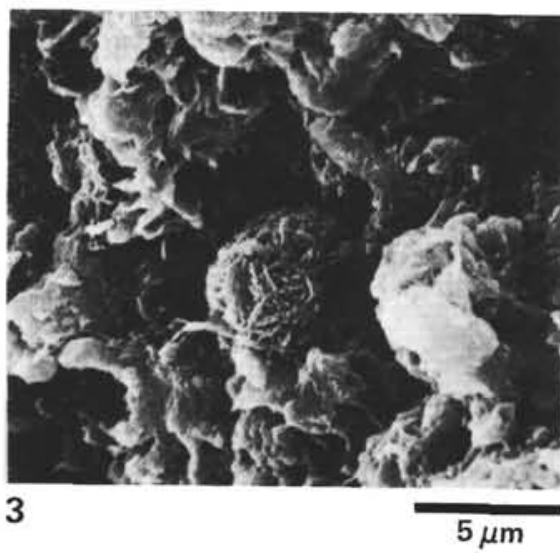

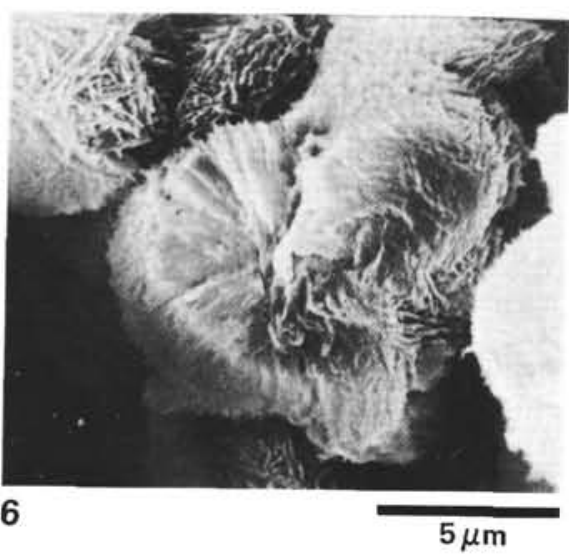

Plate 2. Opal-CT lepispheres in pore spaces of fossils and matrix. 1. Sample 548A-21-1, 56-59 cm (middle Eocene, sub-bottom depth $396 \mathrm{~m}$, $\times 4400$ ), opal-CT lepispheres in a foraminiferal test. 2, 4. Sample 549-32 (top), top-situated nodule (middle Albian?, sub-bottom depth $484 \mathrm{~m}$ ), (2) $\times 1800$, large opal-CT lepispheres in pore space of matrix, (4) $\times 2600$, opal-CT lepispheres in pore space of a foraminiferal test. 3. Sample 551-5-2, 3-5 cm (lower Turonian, sub-bottom depth $134 \mathrm{~m}, \times 5400$ ), opal-CT lepisphere in calcareous matrix. 5. Sample 549-42-2, 38-40 cm (lower Albian, sub-bottom depth $571 \mathrm{~m}, \times 2200$ ), opal-CT lepispheres, euhedral crystal of calcite, and framboidal pyrite crystal aggregates in pore space of clayey siltstone. 6. Sample 549-35-1, 37-39 cm (middle Albian, sub-bottom depth $512 \mathrm{~m}, \times 1500$ ). A section of opal-CT lepispheres can be seen in clayey siltstone matrix. 


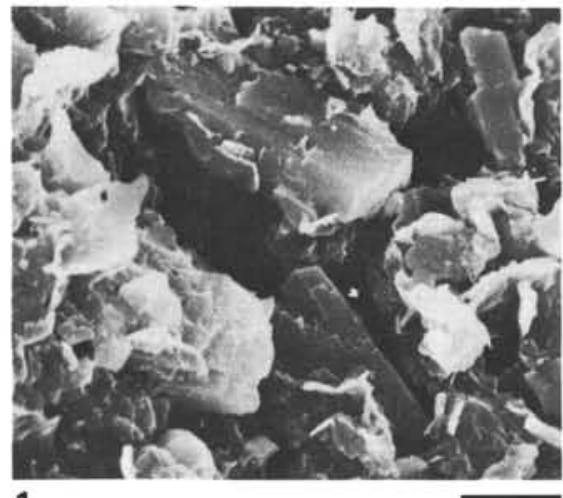

1

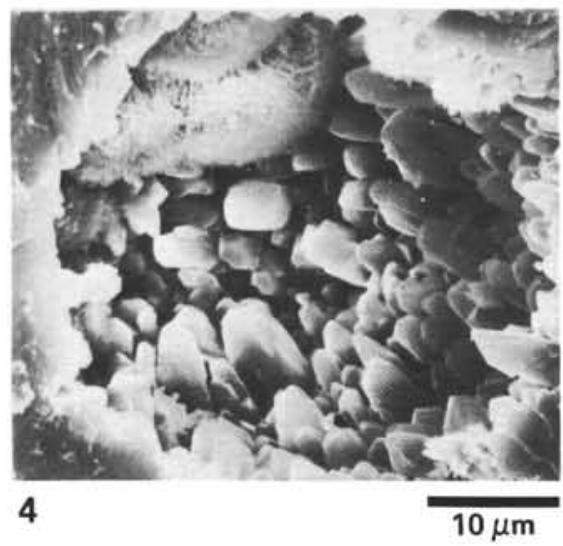

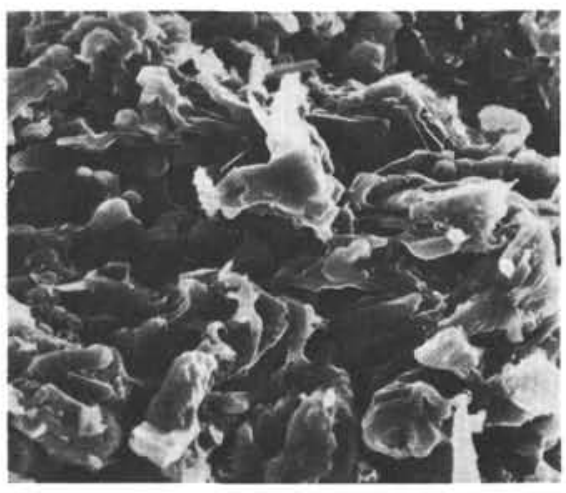

2

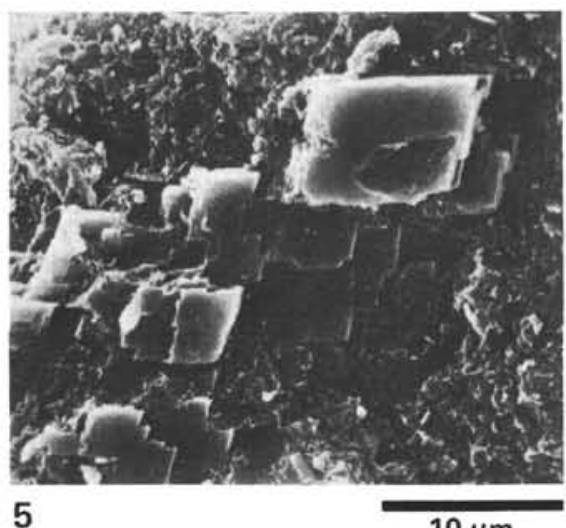

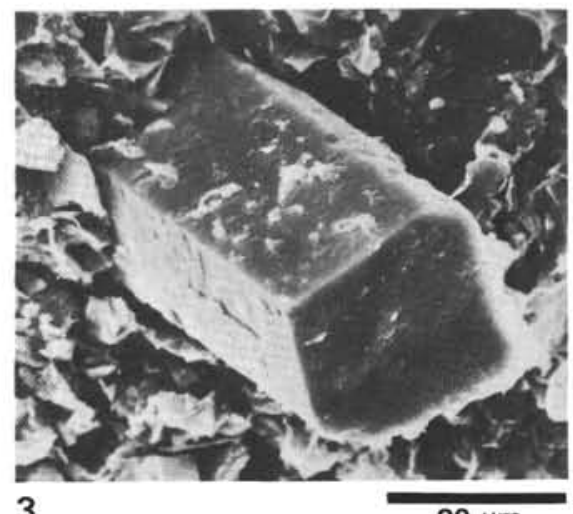

$20 \mu \mathrm{m}$

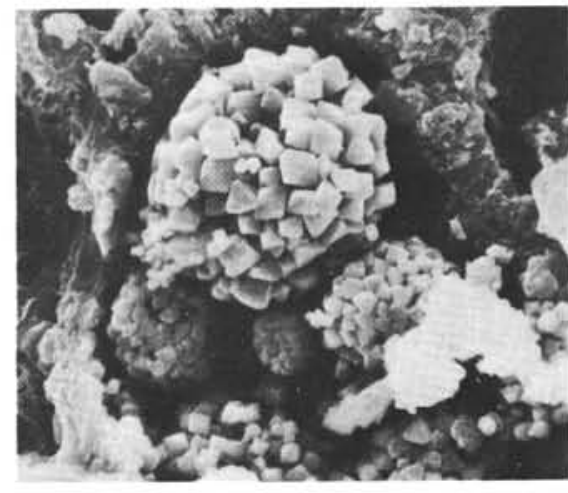

6

$5 \mu \mathrm{m}$

Plate 3. Authigenic zeolites and other diagenetic minerals in silicified sediments. 1-3. Sample 551-5-2, 79-81 cm (lower Turonian, sub-bottom depth $135 \mathrm{~m}),(1) \times 3600$, clinoptilolite crystals in matrix of tuffaceous? chalk, (2) $\times 1260$, dissolved clinoptilolite crystals in matrix of tuffaceous chalk, (3) $\times 1600$, euhedral phillipsite crystal in matrix of clinoptilolite and clay minerals. 4. Sample 549-32 (top), top-situated nodule (middle Albian?, sub-bottom depth $484 \mathrm{~m}, \times 2400$ ), opal-CT lepispheres and authigenic calcite crystals in void of a foraminiferal test. 5. Sample 550-36-1, 132-135 cm (upper Paleocene, sub-bottom depth $424 \mathrm{~m}, \times 3200$ ), dolomite rhombic euhedral crystals in opal CT and clay matrix in dolomitic porcellanite nodule. 6. Sample 548A-21-1, 56-59 cm (middle Eocene, sub-bottom depth $396 \mathrm{~m}, \times 4000$ ), framboidal pyrite crystal aggregates in foraminiferal chamber in porcellanite nodule. The chamber wall has been replaced by opal CT. 